\title{
Nonclassical Ligand-Independent Regulation of Go Protein by an Orphan Class C G-Protein-Coupled Receptor ${ }^{\text {S }}$
}

\author{
Mariana Hajj, Teresa De Vita, Claire Vol, Charlotte Renassia, Jean-Charles Bologna, \\ Isabelle Brabet, Magali Cazade, Manuela Pastore, Jaroslav Blahos, Gilles Labesse, \\ Jean-Philippe Pin, and Laurent Prézeau
}

Institute of Functional Genomics (M.H., T.D.V., C.V., C.R., J.-C.B., I.B., M.C., M.P., J.-P.P., L.P.) and CBS (G.L.), University of Montpellier, French National Center for Scientific Research (CNRS), French National Institute of Health and Medicine (INSERM), Montpellier, France; and Institute of Molecular Genetics, Academy of Sciences of the Czech Republic and Department of Pharmacology, 2nd Medical School, Charles University, Prague, Czech Republic (J.B.)

Received May 16, 2018; accepted May 28, 2019

\section{ABSTRACT}

The orphan G-protein-coupled receptor (GPCR) GPR158 is expressed in the brain, where it is involved in the osteocalcin effect on cognitive processes, and at the periphery, where it may contribute to glaucoma and cancers. GPR158 forms a complex with RGS7- $\beta 5$, leading to the regulation of neighboring GPCR-induced Go protein activity. GPR158 also interacts with $\alpha \mathrm{O}$, although no canonical Go coupling has been reported. GPR158 displays three VCPWE motifs in its C-terminal domain that are putatively involved in G-protein regulation. Here, we addressed the scaffolding function of GPR158 and its VCPWE motifs on Go. We observed that GPR158 interacted with and stabilized the amount of RGS7- $\beta 5$ through a 50-residue region downstream of its transmembrane domain and upstream of the VCPWE motifs. We show that two
VCPWE motifs are involved in $\alpha$ o binding. Using a G $\alpha 0-\beta \gamma$ bioluminescence resonance energy transfer (BRET) sensor, we found that GPR158 decreases the BRET signal as observed upon G-protein activation; however, no constitutive activity of GPR158 could be detected through the measurement of various G-protein-mediated downstream responses. We propose that the effect of GPR158 on Go is unlikely due to a canonical activation of Go, but rather to the trapping of $\mathrm{G} \alpha$ o by the VCPWE motifs, possibly leading to its dissociation from $\beta \gamma$. Such action of GPR158 is expected to prolong the $\beta \gamma$ activity, as also observed with some activators of G-protein signaling. Taken together, our data revealed a complex functional scaffolding or signaling role for GPR158 controlling Go through an original mechanism.

\section{Introduction}

G-protein-coupled receptors (GPCRs) are key players in cellcell communication and the protein family most targeted by commercial drugs (Overington et al., 2006). GPCRs couple to heterotrimeric ( $\alpha \beta \gamma$ subunits) G proteins that control the activity of membrane and intracellular effectors. GPCRs behave as G-protein nucleotide exchanging factors to promote the GTP-bound G-protein $\alpha$ subunit active state, generally considered dissociated from $\beta \gamma$. Furthermore, G-protein $\alpha$ and $\beta \gamma$ activities can be decreased by other signaling proteins, such as regulators of G-protein signaling (RGS) (Gerber et al., 2016) or prolonged by activators of G-protein signaling (AGS) (Blumer and Lanier, 2014). GPCRs also mediate their action

This work was supported by the University of Montpellier through a "Presidence PhD Grant" for M.H.'s PhD training period.

https://doi.org/10.1124/mol.118.113019.

S This article has supplemental material available at molpharm.aspetjournals.org. through G-protein-independent pathways (such as those involving arrestins), signaling cross-talk (Prezeau et al., 2010), receptor transactivation (Milligan, 2006), or association with specific signalosomes (Bockaert et al., 2010). Thus, GPCR signaling pathways must be functionally organized to integrate so many regulatory inputs.

Cellular and physiologic functions of the orphan receptor GPR158 are largely unknown. It is expressed in the brain (Orlandi et al., 2015), where it likely regulates neuron excitability as it has been "fished out" as a partner of potassium Kv4.2 and calcium Cav2 channels (Marionneau et al., 2009; Müller et al., 2010). In the CA3 region of the hippocampus, GPR158 has recently been reported to be involved in the cognitive actions of osteocalcin (Khrimian et al., 2017) and to control the presynaptic differentiation of mossy fiber-CA3 synapses by interacting with proteoglycans of the extracellular matrix (Condomitti et al., 2018). GPR158 could also be involved in the shaping of retinal

ABBREVIATIONS: 7TM, seven transmembrane; AGS, activator of G-protein signaling; BRET, bioluminescence resonance energy transfer; ECD, extracellular domain; ELISA, enzyme-linked immunosorbent assay; FRET, time-resolved Förster resonance energy transfer; GPCR, G-proteincoupled receptor; HEK-293, human embryonic kidney cell line 293; HA, human influenza hemagglutinin; HRP, horseradish peroxidase; HTRF, homogeneous time-resolved FRET; IP1, inositol monophosphate; IP3, inositol phosphate second messengers; mGlu, metabotropic glutamate; MOR, $\mu$-opioid receptor; PDE, phosphodiesterase; PTX, pertussis toxin; RGS, regulatory of G-protein signaling; TR-FRET, time-resolved Förster resonance energy transfer; WT, wild-type. 
photoreceptor signaling (Orlandi et al., 2012). Its expression is regulated by glucocorticoids in trabecular meshwork cells, a mechanism possibly involved in the occurrence of glaucoma (Patel et al., 2013). Although its role in prostate cancer emerged recently (Patel et al., 2015), the impact of GPR158 mutations in colorectal cancers and leukemia must still be confirmed (Wood et al., 2007; Greif et al., 2011). Together, these data suggest important regulatory roles in cellular processes, highlighting the real necessity to understand GPR158's signaling roles in healthy and disease cells.

Potential signaling functions for GPR158 are supported by the observation that GPR158 can interact with the RGS7 protein and the G-protein $\alpha$ o subunit (Orlandi et al., 2012, 2015). Indeed, GPR158 brings RGS7 to the plasma membrane, where RGS7 can allosterically accelerate the GTPase activity of $\mathrm{G} \alpha \mathrm{o}$ and turn it off when Go is activated by neighboring GPCRs. Three short VCPWE motifs are present in the last third of the C-terminal domain of GPR158. Although they resemble the ICPWE motif of the retinal phosphodiesterase (PDE) $\gamma$ subunit known to mediate the interaction with R7 family RGS proteins and active G- protein $\alpha$ subunits (Slep et al., 2001), they were not involved in RGS7 interaction with GPR158 (Orlandi et al., 2015). Thus, whereas RGS7 would interact in the first third of the C-terminal domain of GPR158, G $\alpha$ o may bind on two sites, one in the same region as RGS7 and another in the second half of the C-terminal domain. Surprisingly, the role of the VCPWE motifs remains unclear. Furthermore, GPR158 possesses residues signatures reported to be important for G-protein coupling of other GPCRs (Bjarnadóttir et al., 2005), but the ability of GPR158 to couple to $G$ proteins in a canonical way has not been addressed either. Thus, it seems that GPR158 plays important roles in the absence of ligand, notably through its association with RGS7. The question remains of what the scaffolding role of GPR158 toward RGS7 and Go could be in the absence of ligand.

Here we identified the interaction site of RGS7 in the 714764 region of GPR158 C-terminal domain and confirmed that the three VCPWE motifs are not involved in this interaction. In contrast, these motifs were required for $\mathrm{G} \alpha 0$ association. We also show that GPR158 likely induced G $\alpha o-\beta \gamma$ dissociation as measured by a BRET assay, reflecting possible activation by the receptor; however, we found no evidence of canonical coupling of WT and GPR158 mutants to G protein under basal condition using second-messenger functional assays. Indeed, the GPR158-induced increased levels of dissociated Go is possibly due to trapping of $\mathrm{G} \alpha \mathrm{o}$ by the GPR158 VCPWE motifs. By trapping G $\alpha 0$, GPR158 is expected to prolong the action of $\beta \gamma$, as observed with some group II AGS proteins. These data, which do not exclude the possible direct G-protein activation upon ligand activation of GPR158, unravel new ways for GPCRs to locally regulate G-protein pathways.

\section{Materials and Methods}

Compounds. All compounds and reagents were purchased from the most appropriate sources and companies.

Plasmids and Site-Directed Mutagenesis. To generate C-terminal Flag- or HA-tagged GPR158 constructs, HA or Flag tag sequences were introduced downstream of GPR158 coding region after a unique MluI restriction site, followed by a stop codon. To generate the C-terminal truncated forms ( $\Delta \mathrm{C} 1$ to $\Delta \mathrm{C} 11)$ of GPR158 receptor, a second MluI site was inserted at the required positions, and the DNA sequence between the two MluI sites was excised. To generate $\mathrm{N}$-terminal tagged Ha- and Flag-GPR158 constructs, MluI and $\mathrm{XbaI}$ restriction sites were inserted by polymerase chain reaction after the $\mathrm{N}$-terminal peptide signal and after the C-terminal stop codon of the GPR158 coding sequence, respectively. The resulted MluI-XbaI fragment was then inserted into pRK-HA- and pRK-FlagGABA $_{\mathrm{B} 1}$ (GB1) plasmids (Kniazeff et al., 2004; Rives et al., 2009) digested using the MluI and XbaI sites, which excised the GABA $_{\mathrm{B} 1}$ coding sequence. We generated HA-Snap-GPR158 and Flag-ClipGPR158 by inserting the sequence encoding Snap tag and Clip tag at MluI site of HA-GPR158 and Flag-GPR158, respectively, using a Quick-Change strategy (Stratagene, San Diego, CA). The RGS7 (s2 form) and $\beta 5$ plasmids were purchased from (UMR cDNA Resource Center, MO). The HA tag was introduced at the C-terminal end of the coding sequence of RGS7 upstream of both a XhoI restriction site and a stop codon using a Quick-Change strategy. To generate $\mathrm{GABA}_{\mathrm{B} 1 \mathrm{a}} \mathrm{HA}$, the HA tag sequence was inserted at the C-terminal end of the $\mathrm{GABA}_{\mathrm{B} 1 \mathrm{a}}$ coding sequence between a XhoI site and the stop codon using a Quick-Change strategy. Chimeras formed by exchanging domain between metabotropic glutamate (mGlu)1a and GPR158 or mGlu2 and GPR158 were generated using polymerase chain reaction overlap strategies, and point mutations in GPR158 sequence were generated using the Quick-Change strategy. All final constructs were verified by sequencing (MWG, Ebersberg, Germany).

Cell Culture and Transfection. Human embryonic kidney 293 cell cultures (from American Type Culture Collection) were cultured in Dulbecco's modified Eagle's medium (Invitrogen Life Technologies, Gaithersburg, MD), supplemented with $10 \%$ fetal calf serum (Sigma, L'isle-D'Abeau, Saint-Quentin Fallavier, France), were checked each month for mycoplasma. The cells were seeded in $100-\mathrm{mm}$ plates and incubated at $37^{\circ} \mathrm{C}$ in a $\mathrm{CO}_{2}$ incubator. Cells were transiently transfected using LipofectAMINE 2000 according to the manufacturer's protocol (Thermo Fisher Scientific, Waltham, MA). Cells were seeded in 96-well plates (Greiner Bio-One, Frickehausen, Germany) at 50,000 or 100,000 cells per well. Alternatively, cells were transfected by electroporation as previously described (Maurel et al., 2004). Ten million cells were electroporated with indicated plasmids containing the coding sequence of the proteins of interest and completed to a total amount of $10 \mu \mathrm{g}$ of plasmid DNA with pRK6 empty vector before being plated in 96 -well plates. All media used for cell culture were purchased from Life Technologies/Thermo Fisher Scientific.

Extracellular and Intracellular Antibody Time-Resolved Förster (TRF) Resonance Energy Transfer Assay (Homogeneous TRF). Based on a luminescence resonance energy transfer technology, time-resolved Förster resonance energy transfer (TR-FRET) experiments using labeled antibodies were performed in 96-well plates in homogeneous conditions (HTRF), as previously described (Maurel et al., 2008). Twenty-four hours after transfection, cells were washed with cold Tris-Krebs buffer $(20 \mathrm{mM}$, Tris $\mathrm{pH} 7.4$, $118 \mathrm{mM} \mathrm{NaCl}, 5.6 \mathrm{mM}$ glucose, $1.2 \mathrm{mM} \mathrm{KH}_{2} \mathrm{PO}_{4}, 1.2 \mathrm{mM} \mathrm{MgSO}$, $4.7 \mathrm{mM} \mathrm{KCl}$, and $1.8 \mathrm{mM} \mathrm{CaCl}_{2}$ ) and incubated overnight at $4^{\circ} \mathrm{C}$ with lumiphore-conjugated antibodies (Europium cryptate-labeled antibody, $3 \mathrm{nM}$, and d2- labeled antibody, $6 \mathrm{nM}$ ) (Cisbio Bioassays, Codolet, France). The cells were then incubated for 5 minutes at room temperature with $\mathrm{KF}(200 \mathrm{mM})$. The fluorescence of the europium cryptate $(620 \mathrm{~nm})$ and $\mathrm{d} 2(665 \mathrm{~nm})$ was measured 50 microseconds after excitation at $337 \mathrm{~nm}$ using RubyStar or PHERAStar plate readers (BMG Labtechnologies, Champigny-surMarne, France). The TR-FRET signals were expressed as $\% \Delta \mathrm{F}=$ (665/620)sample-(665/620)mock*100/(665/620)mock. For the intracellular antibody TR-FRET assay, measurements were performed in cells permeabilized with Triton X-100 $0.02 \%(\mathrm{v} / \mathrm{v})$ before overnight incubation at $4^{\circ} \mathrm{C}$ with lumiphore-conjugated antibodies (europium cryptate-labeled antibody $(3 \mathrm{nM})$ and $\mathrm{d} 2$ - labeled antibody $(6 \mathrm{nM}))$ (Cisbio Bioassays). 
Cell-Surface Protein-Level Quantification by Enzyme-Linked Immunosorbent Assay and SNAP Labeling. Enzyme-linked immunosorbent assay (ELISA) experiments were performed as previously described (Maurel et al., 2008). Briefly, cells were fixed with $4 \%$ paraformaldehyde and blocked with phosphate-buffered saline (PBS) containing $1 \%$ fetal calf serum and then incubated 30 minutes at $0.5 \mathrm{mg} /$ /iter with monoclonal anti-Flag M2 antibodies (Sigma), anti-HA antibodies (clone 3F10; Roche Applied Science, Basel, Switzerland) or anti-Myc antibodies (clone 9E10; University of Iowa, Iowa City, IA). When these primary antibodies were not conjugated themselves with horseradish peroxidase (HRP), cells were further washed and incubated (30 minutes) with HRP-conjugated goat anti-rat IgG $(0.5 \mathrm{mg} / \mathrm{liter}$; Jackson ImmunoResearch Laboratories, Westgrove, PA) or anti-rabbit IgG or anti-mouse IgG ( 0.5 mg/liter; Amersham Biosciences GE Healthcare, Chicago, IL) for 30 minutes. After washes, bound antibody was detected by chemiluminescence using SuperSignal substrate (Pierce, Rockford, IL) and a Mithras LB 940 plate reader (Berthold Biotechnologies, Bad Wildbad, Germany). As a control for intracellular ELISA quantification, cells were permeabilized for 5 minutes with $0.05 \%$ Triton X-100 just after being fixed. SNAP-tag labeling was performed as described previously (Doumazane et al., 2011). Briefly, 24 hours after transfection, human embryonic kidney 293 cells (HEK293) cells were incubated at $37^{\circ} \mathrm{C}$ for 1 hour with a solution of $100 \mathrm{nM}$ of Lumi4-Tb (Cisbio Bioassays). After labeling, the cells were washed three times with Krebs buffer, and drugs were added as described. TR-FRET measurements were performed on INFINITE 500 (TECAN, Männedorf, Switzerland) or PHERAstar FS (BMG Labtechnologies) microplate readers, which are equipped a standard with TR-FRET optical modules.

Coimmunoprecipitation and Western Blotting. At 48 hours after transfection in 100-mm plates, cells were washed with ice-cold PBS-GAB (PBS supplemented with glucose and antibiotics). After washes, cells were scraped with PBS-GAB and centrifuged for 5 minutes at $2000 \mathrm{rpm}$. The supernatant was removed, and the pellet was resuspended in lysis buffer [Hepes $1 \mathrm{M}$, NaCl $5 \mathrm{M}$, NP40 20\%, glycerol, dodecyl maltoside, and protease inhibitors cocktail (Sigma)]. The lysate was incubated for 2 hours at $4^{\circ} \mathrm{C}$ with mild shaking and then clarified by centrifugation at $15,000 \mathrm{~g}$ for 15 minutes. Clarified lysate was incubated with monoclonal anti-HA conjugate agarose beads, (Clone HA-7; Sigma) overnight at $4^{\circ} \mathrm{C}$ and then centrifuged for 2 minutes at $13,000 \mathrm{rpm}$. The supernatant was removed, and the pelleted beads were washed four times with PBS $1 \times$ before elution by addition of loading buffer. The samples were loaded on NuPAGE Novex 3\%-8\% Tris-acetate midi gel (Invitrogen Life Technologies) transferred to nitrocellulose membrane (Amersham Biosciences GE Healthcare, Chicago, IL) and subjected to immunoblotting. The primary rabbit anti-HA (Invitrogen Life Technologies) antibody was used at $0.6 \mathrm{mg} /$ liter and the mouse anti-Flag antibody (F3165; Sigma) at $2 \mathrm{mg} /$ liter. Secondary antibodies anti-rabbit HRP-linked IgG (0.5 mg/liter; Amersham Biosciences GE Healthcare) and antimouse HRP-linked IgG (Santa Cruz, Dallas, TX) were applied for 30 minutes. Immunoreactive bands were visualized by ECL detection kit (Amersham Biosciences GE Healthcare) on Kodak ML light films.

IP-One and cAMP Assays. Experiments were performed in a 96-well plate format. The IPOne HTRF kit (Cisbio Bioassays) was used according to the recommendations of the manufacturer to measure the production of inositol phosphate second messengers (IP3) through assessment of inositol monophosphate (IP1) accumulation, a downstream metabolite of IP3. Cells were incubated in the presence of indicated receptor agonist for 30 minutes at $37^{\circ} \mathrm{C}$ and then incubated in the presence of a cryptate-labeled anti-IP1 or cAMP antibodies and D2-labeled IP1 or cAMP for 1 hour at room temperature. The fluorescence of the europium cryptate and $\mathrm{d} 2,620$, and $665 \mathrm{~nm}$ respectively, was measured (without washing) 50 microseconds after excitation at $337 \mathrm{~nm}$ using RubyStar or PHERAstar plate readers (BMG Labtechnologies).

BRET Experiments. As previously described (Ayoub et al., 2007) for saturation curves, a constant amount of a plasmid encoding one of the proteins of interest bearing the donor-Luc was coexpressed with a range of expression of the plasmid encoding the second protein of interest bearing the acceptor-YFP. After washing of the cells, the Luciferase substrate Coelenterazine h (cat. no. C-6780 Invitrogen/Thermo Fisher Scientific) was added ( $5 \mathrm{mM}$ in $50 \mu \mathrm{l}$ per well) to initiate the BRET process. Readings were recorded using the Mithras LB940 reader (Berthold Biotechnologies) (Rluc filter: $485 \pm 20 \mathrm{~nm}$ and YFP filter: $530 \pm 25 \mathrm{~nm}$ ), data were collected using the MicroWin2000 software, and BRET signal expressed in milliBRET units of BRET ratio. Dose-response curves were fitted with a linear regression or sigmoid dose-response equation, using Prism (GraphPad software, San Diego). The $\mathrm{G} \alpha_{\mathrm{o}}$ experiments were performed as previously described (Rives et al., 2009). The Go activation/dissociation BRET assays were performed as described previously (Brulé et al., 2014). The transfected HEK293 cells were washed with PBS, and readout was performed on a Mithras LB940 plate reader (Berthold Technologies) at $37^{\circ} \mathrm{C}$ after the addition of cœlenterazine $\mathrm{h}(5 \mu \mathrm{M})$ and ligand. When indicated, treatment with pertussis toxin $(100 \mathrm{ng} / \mathrm{ml})$ was performed for 16 hours before stimulation of the cells. The BRET ratio was calculated based on the difference between the emission at $530 / 485 \mathrm{~nm}$ of co-transfected Rluc and YFP fusion proteins and the emission at 530/485 $\mathrm{nm}$ of the Rluc fusion protein alone.

Data Analysis and Statistical Tests. Data were analyzed using Prism 7.0e (GraphPad) or Excel 16.16.8 (Microsoft), and statistical tests were performed using Prism tutorial and $\mathrm{R}$ software.

\section{Results}

GPR158 Forms Homodimers at the Cell Surface. The orphan receptor GPR158 shares homology within its seventransmembrane (7TM) domain with the class C GPCRs (Bjarnadóttir et al., 2005), including the mGlu and GABA $\left(\mathrm{GABA}_{\mathrm{B}}\right)$ receptors. Like most class C GPCRs, GPR158 also has a large N-terminal extracellular domain linked to the 7TM domain via a cysteine-rich domain; however, neither part of the N-terminal domain is related to those of the class C GPCRs (Kniazeff et al., 2011). The N-terminal part of most class C GPCRs, called the Venus flytrap domain, contains the agonist binding site and is responsible for the constitutive dimerization of these receptors (Pin and Bettler, 2016; Koehl et al., 2019).

We observed that, like most class C GPCRs, GPR158 also exists as homodimers at the surface of transfected HEK293 cells. Indeed, Western blots showed two major bands possibly corresponding to GPR158 monomers and dimers, regardless of which antibodies were used to reveal the protein (Fig. 1A). Moreover, N-terminally HA epitope-tagged GPR158 (HA-GPR158) coimmunoprecipitated N-terminally Flag epitope-tagged GPR158 (Flag-GPR158) coexpressed in HEK293 cells (Fig. 1B). In contrast, neither Flag-mGlu2 nor the Flag-GB2 subunit of the $\mathrm{GABA}_{\mathrm{B}}$ receptor coimmunoprecipitated with HA-GPR158 receptor (Fig. 1B), whereas HA-GB2 association to its Flag-GB1 subunit partner could easily be detected (Fig. 1B). Cellular dimeric TR-FRET signals (Maurel et al., 2008) were detected using anti-HA and anti-Flag antibodies labeled with luminescence resonance energy transfer-compatible dyes in cells coexpressing HA-GPR158 and Flag-GPR158, but not in cells coexpressing Flag-GPR158 and HA-GB2 (Fig. 1, C and D). Finally, Flag-GPR158 homodimer formation was disrupted by an increasing amount of HA-GPR158, but not by HA-GB2 (Fig. 1E). Taken together, these data indicate that GPR158, like the other class C GPCRs, forms homodimers at the cell surface. Given that stoichiometry is a mandatory feature of functional class C GPCRs (El Moustaine et al., 2012), we 
A

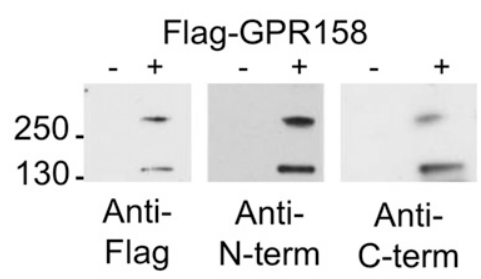

B

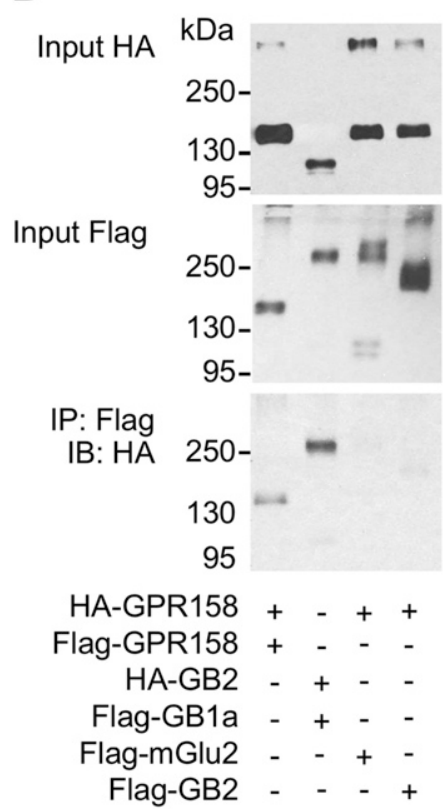

C

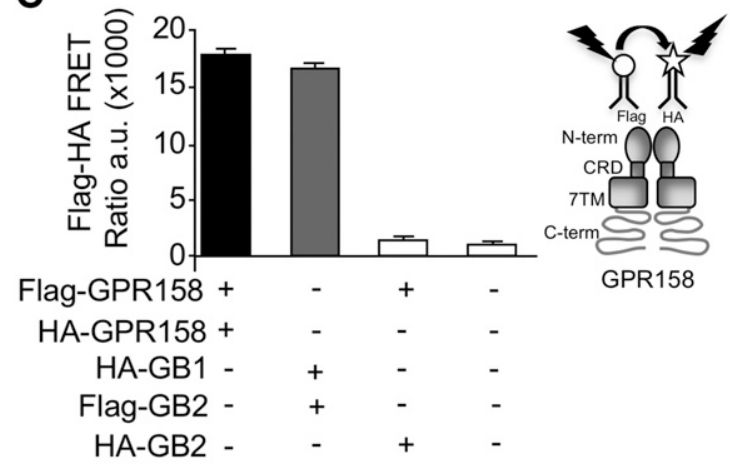

D

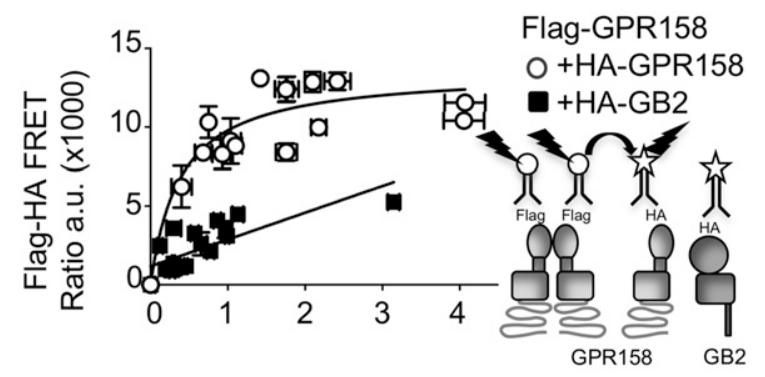

E

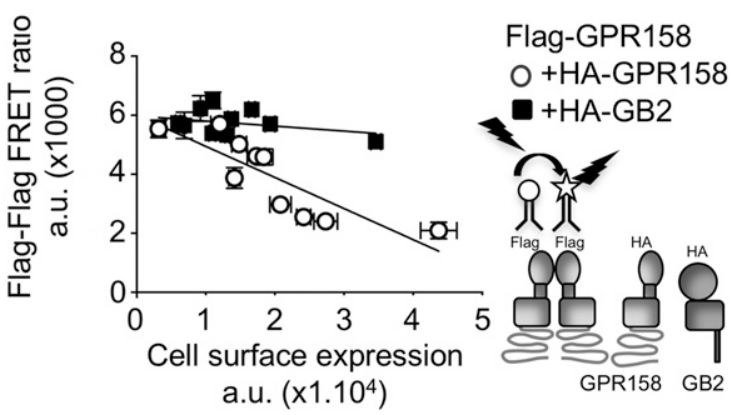

Fig. 1. GPR158 forms dimers at the cell surface. (A) Western blot analysis using three different commercially available antibodies, recognizing the Flag epitope (M2 clone), the N-terminal (Anti N-term, SAB4502509 Sigma), or the C-terminal domain (anti C-term, HPA013185 Sigma) of N-terminal Flagtagged GPR158 (Flag-GPR158), revealed two major bands, at around 150 and $300 \mathrm{kDa}$, in HEK293 cells transiently expressing Flag-GPR158 (+), but not in mock-transfected cells (-). (B) Coimmunoprecipitation of Flag- and HA-GPR158 coexpressed in HEK293 cells. Note that HA-GPR158 did not coimmunoprecipitate with N-terminal-tagged Flag-mGlu2 or Flag-GB2 $\left(\mathrm{GABA}_{\mathrm{B} 2}\right)$, whereas a positive control showed a coimmunoprecipitation between the two subunits Flag-GB1a and HA-GB2 of the dimeric GABA $A_{B}$ receptor. (C) TR-FRET analysis of GPR158 dimerization. A TR-FRET signal was recorded between Flag- and HA-GPR158, using TR-FRET donor and acceptor fluorophores labeled anti-Flag and anti-HA antibodies. Similarly, a strong

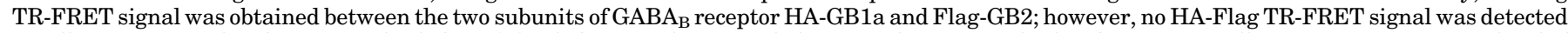
in cells expressing Flag-GPR158 and HA-GB2. (D) HA-GPR158, but not HA-GB2, can dimerize with Flag-GPR158. In cells expressing a constant level of Flag-GPR158, the Flag-HA TR-FRET signal increased when the amount of HA-GPR158 increased up to saturation, in contrast to HA-GB2 increasing expression. (E) HA-GPR158, but not HA-GB2, competed for dimerization with Flag-GPR158. In cells expressing a constant amount of Flag-GPR158, an increasing amount of HA-GPR158 decreased Flag-Flag TR-FRET signal, indicating a competition in the dimer formation between the Flag- and the HA- versions of GPR158, whereas no competition was observed when coexpressing an increasing amount of HA-GB2. In (A-C) panels, data are representative of three independent experiments. In (D and E), data from three independent experiments are pooled. Data are means \pm S.E.M. of triplicate determinations. $\mathrm{N}$ - and C-terminal stand for $\mathrm{N}$ - and C-terminal domain, respectively.

analyzed any possible functional role of GPR158. We first further explored the role of its intracellular domain already reported to interact with G-protein-mediating signaling proteins, including Go and RGS7.

RGS7 Protein Level Is Stabilized by a Specific Region of GPR158 C-Terminal Domain. In transiently transfected HEK293 cells, GPR158, but not the related GB2 $\mathrm{GABA}_{\mathrm{B}}$ subunit, interacted with RGS7 and not with RGS4, as shown by coimmunoprecipitation (Fig. 2, A and B). In these experiments the G-protein subunit $\beta 5$ was coexpressed with RGS7 as it forms a stable complex with RGS7 and protects it from proteolytic degradation (Anderson et al., 2009)
(Supplemental Fig. 1A). The RGS7: $\beta 5$ interaction with GPR158 was confirmed by a TR-FRET approach (Supplemental Fig. 1B). Indeed, a large TR-FRET signal was measured in cells expressing C-terminally Flag-tagged GPR158 (GPR158-Flag) and RGS7-HA, incubated with TR-FRET compatible anti-HA or anti-Flag antibodies, after cell permeabilization. Interestingly, no signal was observed between GPR158 and RGS4-HA. The differential signal measured between RGS7-HA and RGS4-HA expressing cells was not due to different levels of these two proteins nor to a differential amount of GPR158 (Fig. 2A; Supplemental Fig. 1B). 
A
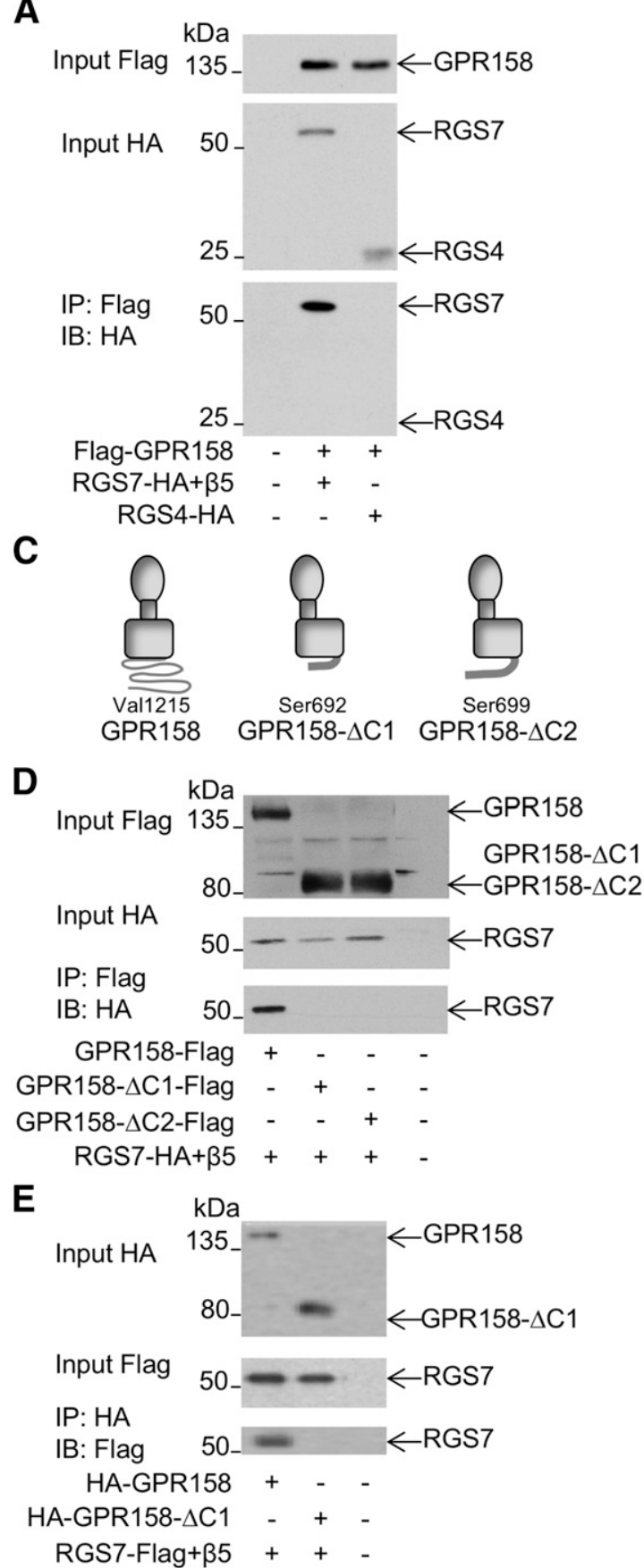

B

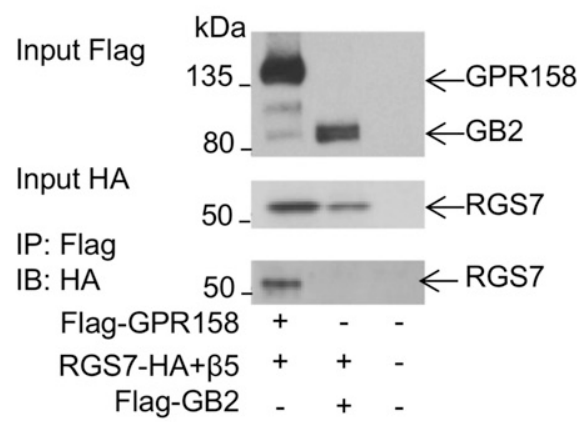

$\mathbf{F}$
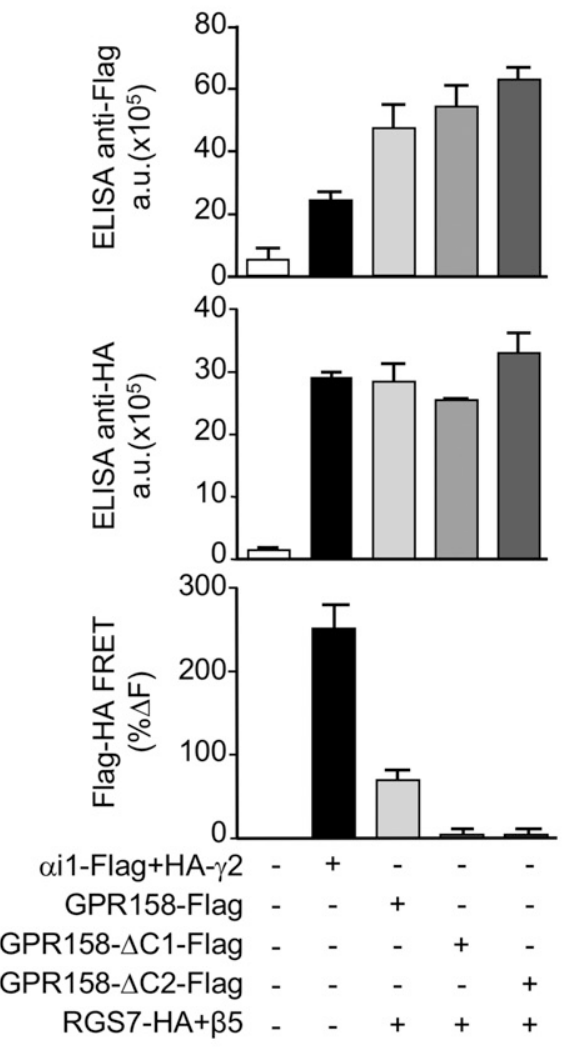

Fig. 2. GPR158 C-terminal domain is required for RGS7 interaction. (A) C-terminal HA-tagged RGS7 (RGS7-HA), but not the C-terminal tagged RGS4 (RGS4-HA) was coimmunoprecipitated by Flag-GPR158 when coexpressed in HEK293 cells. (B) Flag-GPR158, but not Flag-GB2, was coimmunoprecipitated with RGS7-HA in transfected HEK293 cells. (C) Schematic representation of the WT and truncated $\Delta \mathrm{C} 1$ and $\Delta \mathrm{C} 2$ (with the last residues mentioned) versions of GPR158 receptor. (D-F) RGS7 binds to the C-terminal domain of GPR158, as shown with coimmunoprecipitation (D and E) and TR-FRET (F) approaches. HEK293 cells were transfected with WT or C-terminal domain truncated versions $\Delta$ C1 and $\Delta$ C2 of GPR158 together with RGS7 and $\beta 5$. In (D), the coimmunoprecipitation was performed using the C-terminal Flag-tagged versions of the WT (GPR158-Flag) and truncated (GPR158$\Delta$ C1-Flag, GPR158- $\Delta$ C2-Flag) GPR158 receptor, coexpressed in HEK293 cells with RGS7-HA. In (E), the coimmunoprecipitation was performed using HA-GPR158 or HA-GPR158- $\triangle$ C1, coexpressed in HEK293 cells with the C-terminal Flag-tagged RGS7 (RGS7-Flag). (F) For TR-FRET experiments, HEK293 cells expressing truncated or WT GPR158-Flag and RGS7-HA were permeabilized with tritonX-100 (0.1\%) and incubated with antibodies against HA and Flag epitopes bearing the donor and acceptor fluorophores. Each experiment shown is representative of four independent experiments, and data in $(\mathrm{F})$ are the mean \pm S.E.M. of triplicates.

Using membrane-targeting microscopy and in vitro coimmunoprecipitation assays, Orlandi et al. (2015) reported that RGS7 interacted with the C-terminal domain of GPR158. In agreement with this observation, we found that the C-terminal truncated forms of GPR158, which ended at residues Ser692
(GPR158- $\Delta$ C1) or Ser699 (GPR158- $\Delta$ C2) (Fig. 2C), did not coimmunoprecipitate RGS7 (Fig. 2, D and E). Additionally, no significant intracellular TR-FRET signal with RGS7 could be measured with these cropped GPR158 mutants (Fig. 2F), despite protein levels being similar to those of wild-type 
(Fig. 2, D-F). Unexpectedly, the three conserved VCPWE motifs of GPR158 (Fig. 3A; Supplemental Fig. 2) were not involved in RGS7 binding (Fig. 3, B and C), despite their similarity to the PDE $\gamma$-subunit motif known to participate in complex formation with the RGS7-related RGS9 protein
(Slep et al., 2001). Indeed, mutation of the three motifs individually (GPR158-Mut1, -Mut2, and -Mut3) or in combination (GPR158-Mut4) (Fig. 3A) did not suppress GPR158: RGS7 interaction as measured by either coimmunoprecipitation (Fig. 3B) or TR-FRET (Fig. 3C) approaches.
A
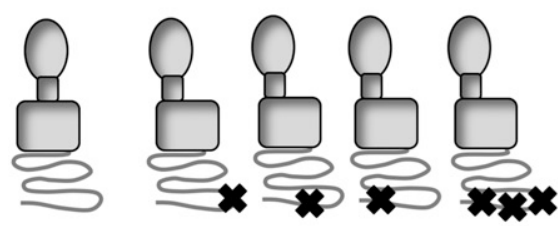
VCPWE VCLWE Mut1 Mut2 Mut3 Mut4 VAAAE VCPWE VCPWE $V A A A E$ VCLWE VAAAE VCLWE VAAAE VCPWE VCPWE VCPWE VAAAE VAAAE
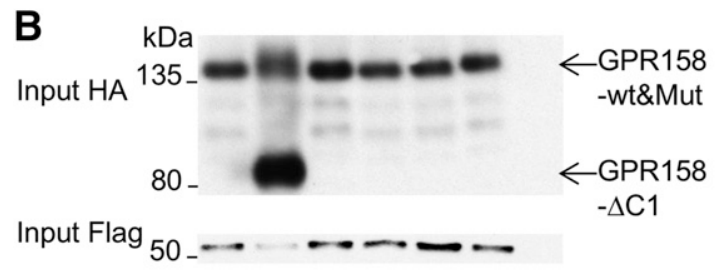

IP: HA

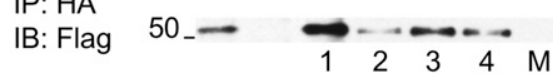

GPR158-HA wt $\Delta \mathrm { C } 1 \longdiv { \text { Mut } }$

RGS7-Flag+ $\beta 5$

D

Truncated GPR158

$\Delta$ C1: Ser692 $\Delta$ C7: Leu914

$\Delta$ C3: lle714 $\Delta$ C8: Ala964

$\Delta$ C4: Thr764 $\Delta$ C9: Leu1014

$\Delta$ C5: Leu814 $\quad \Delta$ C10: Ala1114

$\Delta$ C6: Asp864 $\Delta$ C11: Leu1165

E

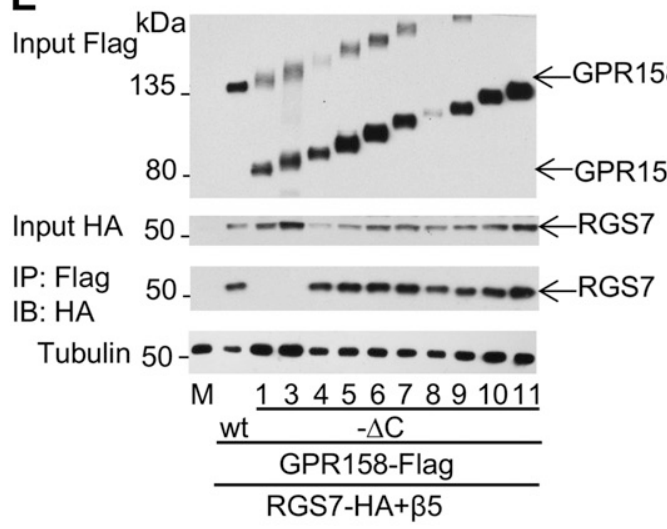

C
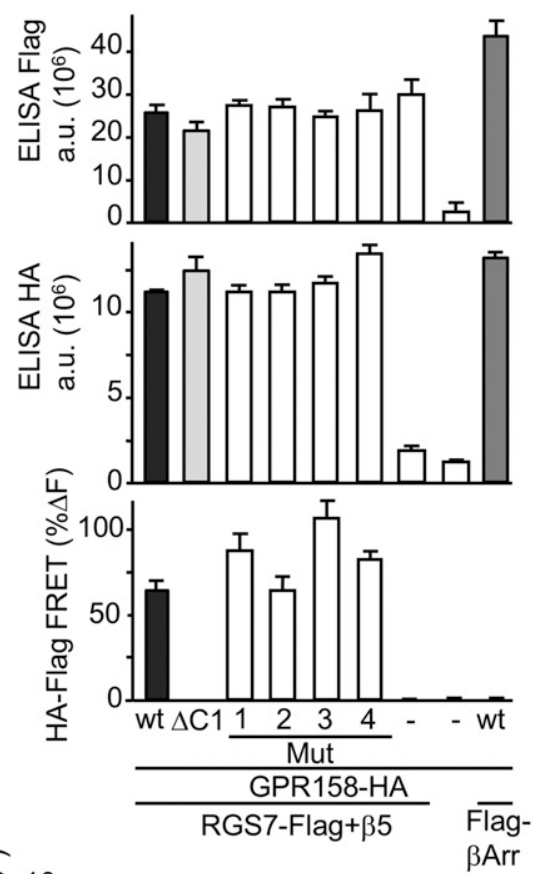

$\mathbf{F}$

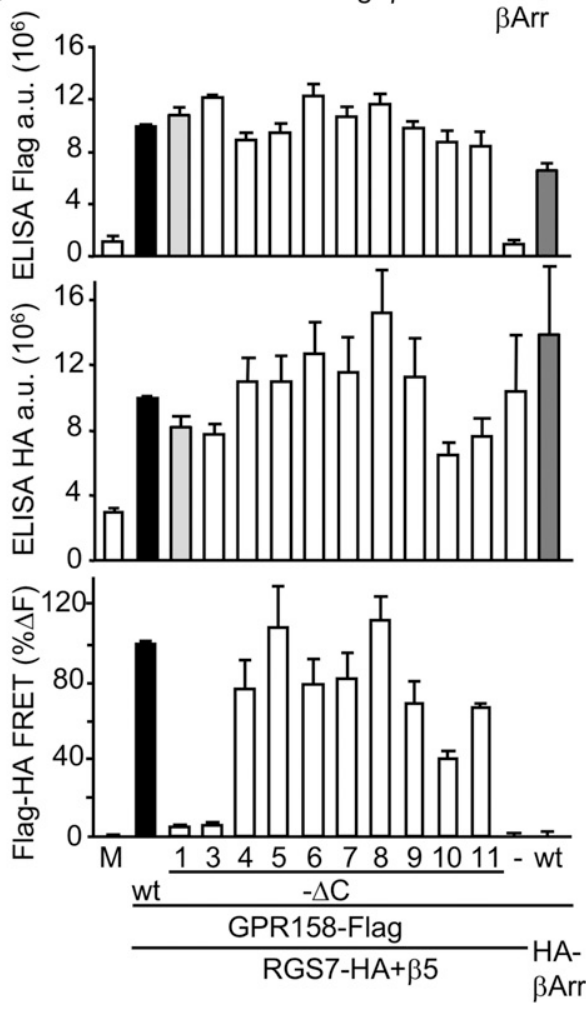

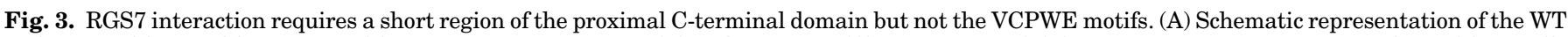

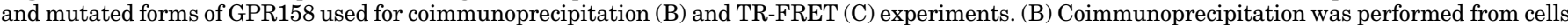

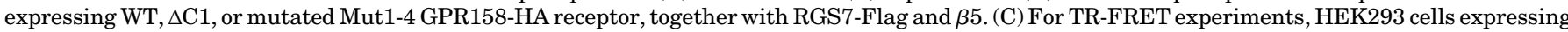

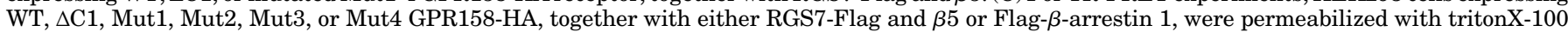

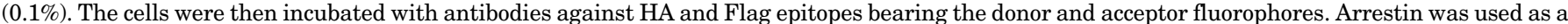

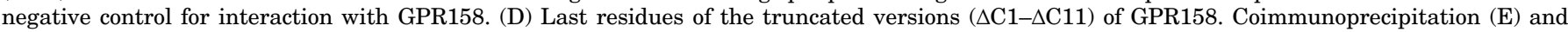

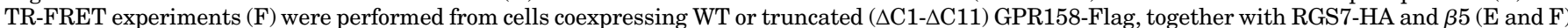

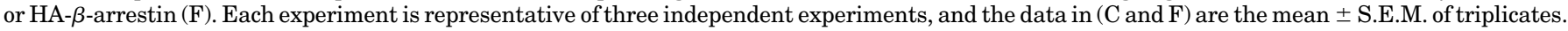


Using a series of GPR158-Flag C-terminal deletion mutants (GPR158- $\Delta \mathrm{C} 1$ to $-\Delta \mathrm{C} 11$, Fig. 3D), we identified the region encompassing residues 714 to 764 as the RGS7 binding site using both coimmunoprecipitation and TR-FRET approaches (Fig. 3, E and F). The 714-764 region overlaps with the region reported by Orlandi et al. (2015) to contribute to RGS7 binding. We found that this GPR158-RGS7 interaction enhanced RGS7 abundance in both the absence or presence of its $\beta 5$ partner (Fig. 4; Supplemental Fig. 3). Using a constant amount of transfected RGS7-coding plasmid, RGS7 protein level was increased in HEK293 cells coexpressing increasing levels of GPR158 (Fig. 4), but not in cells expressing either mGlu2 (Fig. 4A) or GPR158- $\Delta \mathrm{C} 1$ (Fig. 4B). Of note, the expression of mGlu2 actually led to decreased level of RGS7 protein (Fig. 4A). Whether this is due to mGlu2 itself or to its constant activity due to ambient glutamate in cell culture media remains to be clarified. The enhancing effect exerted by GPR158 was less pronounced in the absence of $\beta 5$ (Supplemental Fig. 3). Such a stabilizing effect involves the RGS7 binding domain of GPR158 as revealed using a series of mGlu2 chimeric constructs containing the WT or mutated C-terminal domain of GPR158 (Supplemental Fig. 4, A and B).

These data identified a specific region in the C-terminal domain of GPR158, not including the VCPWE motifs, interacting with and stabilizing RGS- $\beta 5$. We then wondered whether these VCPWE motifs were involved in the interaction with G $\alpha$ o, a RGS7-regulated G-protein subunit that has been reported to interact with the C-terminal domain of GPR158 (Orlandi et al., 2012, 2015).

VCPWE Motifs Contribute to Gao Binding. We first confirmed the interaction between GPR158 and G $\alpha$ o using both TR-FRET (Fig. 5A) and BRET approaches (Fig. 5B). Indeed, the GPR158 and G $\alpha$ o interaction was supported by the generation of a saturating BRET signal curve (Fig. 5B) in HEK293 cells expressing various expression ratios of GPR158-Venus and Goo-RLuc. In contrast, a nonspecific, linear low BRET signal curve was obtained in control cells expressing GPR158-Venus and Homer3-RLuc (Fig. 5B), an $\mathrm{mGlu}$ receptor interacting intracellular protein (Rives et al., 2009). Interestingly, a saturating BRET curve was also observed in cells expressing GPR158-Venus and the G $\alpha 0$ related Goi1-RLuc protein, whereas no BRET signal could be detected with G $\alpha$ q-RLuc (Fig. 5C), suggesting that GPR158 can interact with the G $\alpha \mathrm{i} / \mathrm{o}$ protein family members, but not with $\mathrm{G} \alpha \mathrm{q}$. We then showed that the C-terminal domain of GPR158 was required for this interaction since the TR-FRET signal was greatly reduced in cells expressing G $\alpha 0$-Flag and the truncated GPR158- $\Delta$ C1-HA or GPR158- $\Delta$ C2-HA (Fig. 5A). The VCPWE motifs located in the cytoplasmic domain of GPR158 were identified as key elements for the interaction with $\mathrm{G} \alpha \mathrm{o}$, as the mutation of all three motifs (GPR158-Mut4) largely decreased the TR-FRET signal with G $\alpha$ o (Fig. 5A). Whereas Mut1 and Mut3 show a lower TR-FRET signal than WT GPR158 with G $\alpha$ o, the signal with Mut2 was not affected (Fig. 5A lower panel). These results revealed that motifs 1 and 3 were involved in the association of G $\alpha 0$ with GPR158; however, the Mut1 protein level was significantly reduced compared with that of Mut3 (Fig. 5A, middle panel), suggesting that Mut1 is involved to a lesser extent than is Mut3 in $\alpha 0$ interaction. When using a BRET approach (Fig. 5D), and to a lesser extent using a TR-FRET approach (Fig. 5A), G $\alpha$ o interaction with GPR158-Mut4 could still be measured;
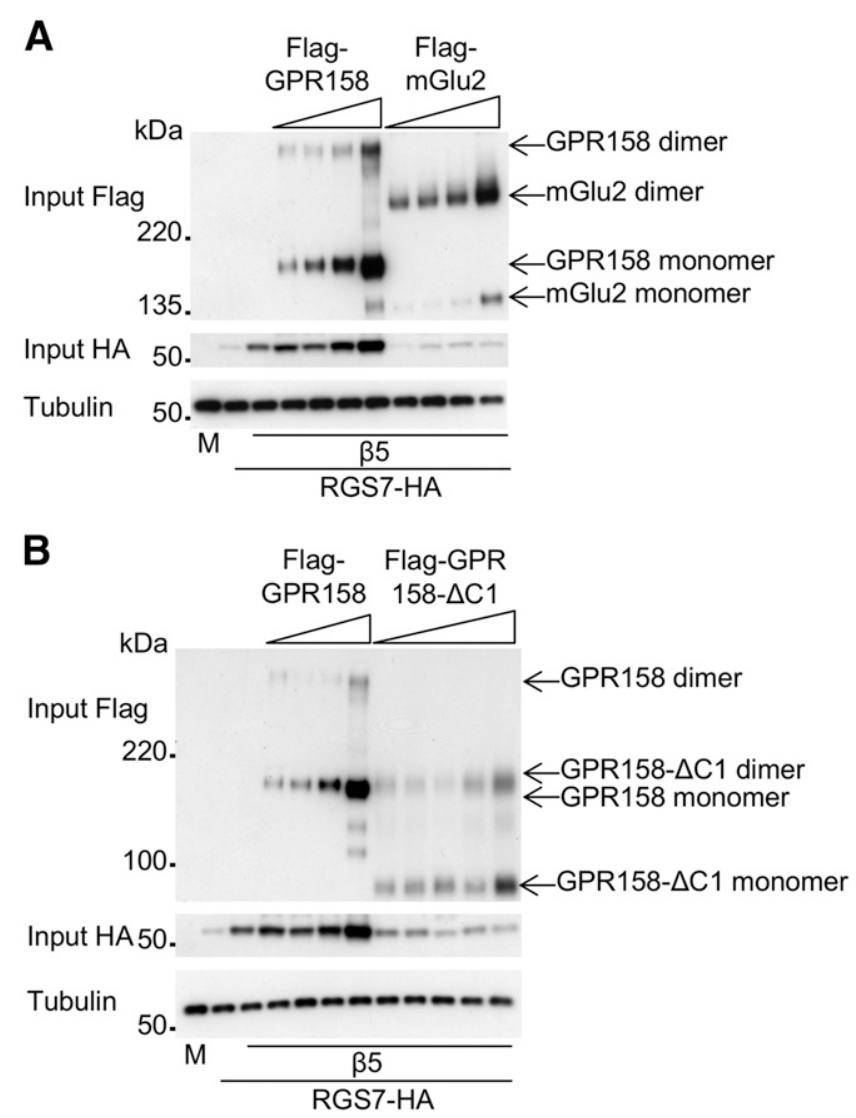

Fig. 4. RGS7 protein level is stabilized when coexpressed with GPR158. HEK293 cells were transfected with constant amounts of plasmids coding for RGS7-HA (150 ng) and $\beta 5(30 \mathrm{ng})$ and increasing amounts of plasmids coding for Flag-GPR158 or Flag-mGlu2 receptors (1-50 ng) (A) or FlagGPR158 or Flag-GPR158- $\Delta$ C1 (1-50 ng) (B). The abundance of HA-RGS7 protein was analyzed using Western blot analysis using an anti- HA antibody. The tubulin protein abundance was used as a Western blot loading control. Each experiment is representative of three independent experiments.

however, the signal was lower than that obtained with the WT GPR158, consistent with the existence of a second G $\alpha$ o interacting site in GPR158 (Orlandi et al., 2015). The difference between the observed TR-FRET and BRET signals is likely related to photophysical properties of the techniques, as the dyes used for each technique display different Ro (5 and $10 \mathrm{~nm}$, respectively). This difference would be enhanced by the larger distance contributed by the size of antibodies used in the TR-FRET approach. Because GPR158 behaves as a scaffolding protein for G-protein signaling proteins and can interact with G-protein subunits, even in absence of ligand, we then asked whether GPR158 displayed GPCR canonical basal coupling to G proteins, as described for many GPCRs (Oh et al., 2006; Thathiah et al., 2009).

GPR158 Did Not Display Detectable Constitutive Coupling to Gq, Gs, or Gi/o Proteins. To assess the putative basal G-protein activation by GPR158, we used a BRET assay. This assay monitors the association state of the G $\alpha 0-\beta \gamma$ protein complex composed of G $\alpha 0-R L u c$ and $\beta \gamma$-Venus. The BRET signal decreases when a Go-coupled GPCR is activated, as illustrated with the $\mu$-opioid receptor (MOR) (Fig. 6A) and the $\mathrm{GABA}_{\mathrm{B}}$ receptor (Fig. 6B). In contrast, the BRET signal was not affected by the 
A
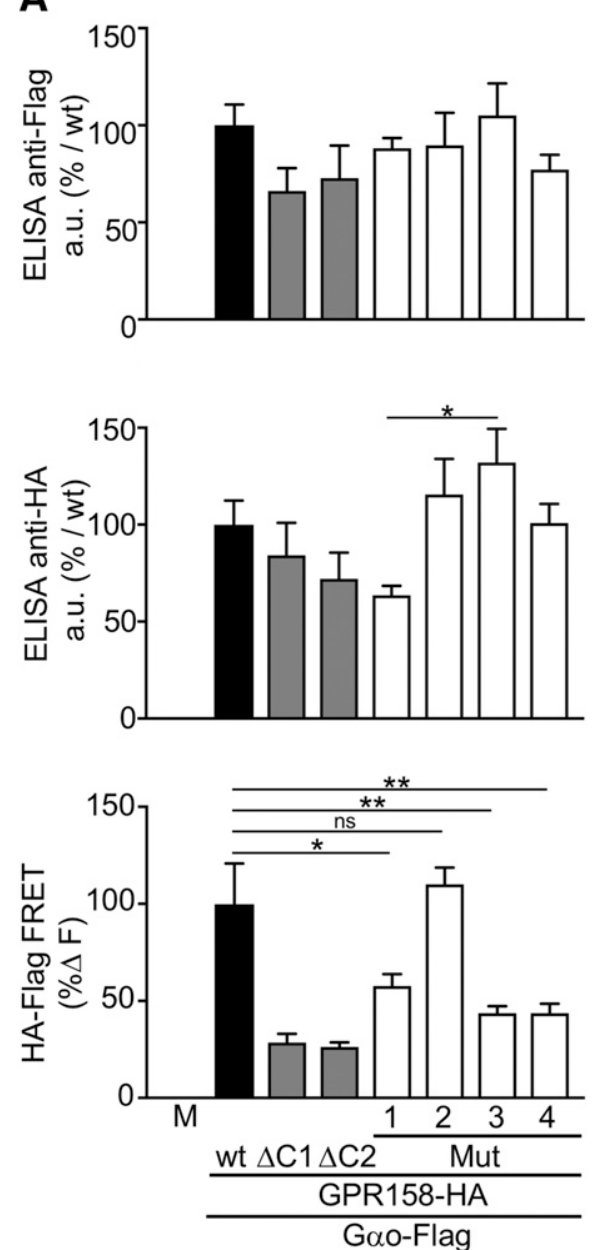

B

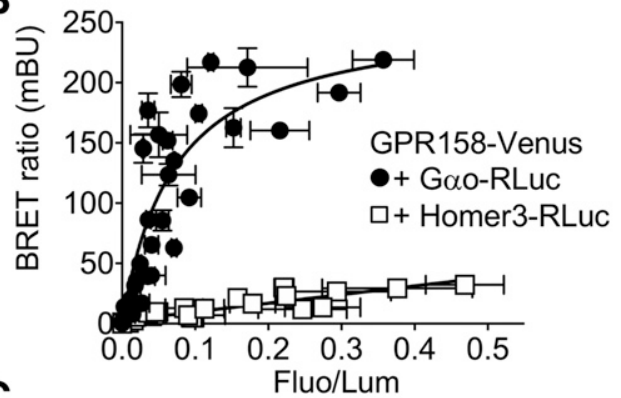

C
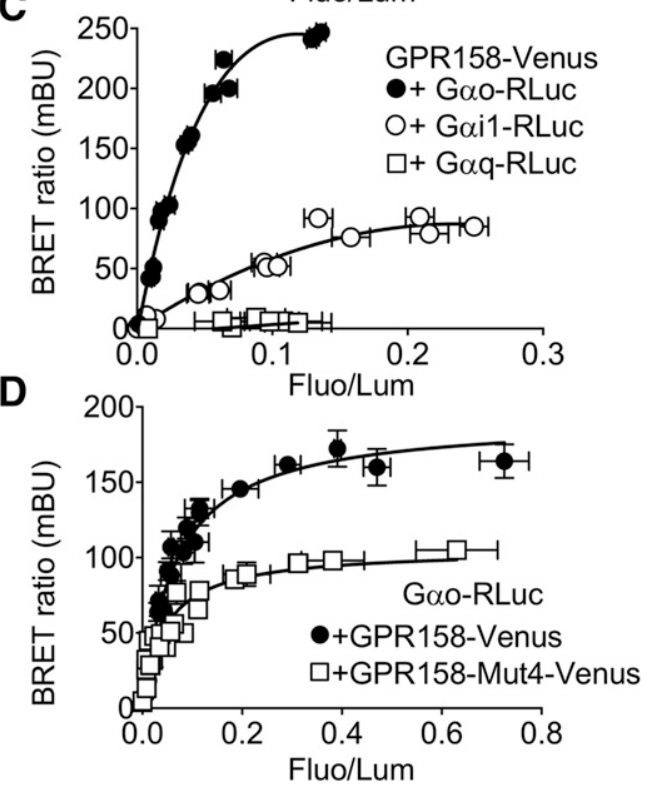

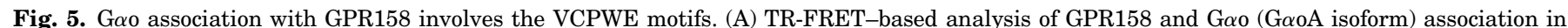

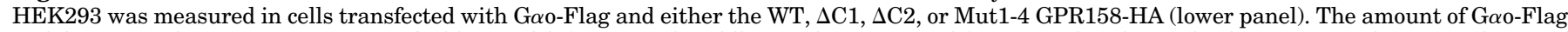

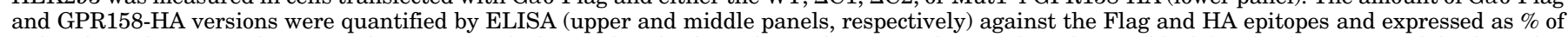

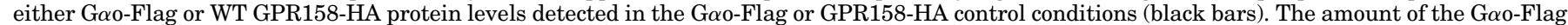

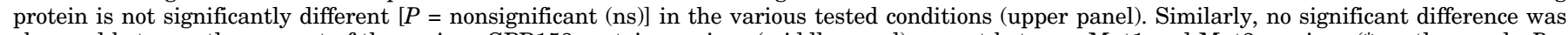

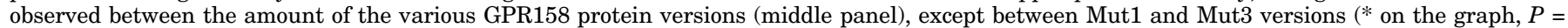

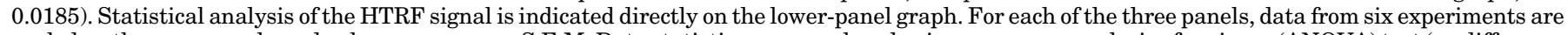

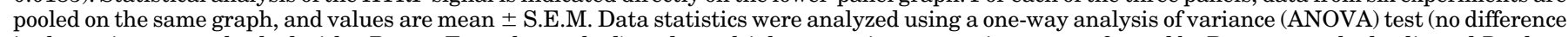

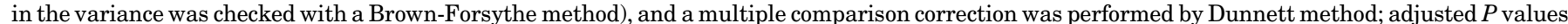

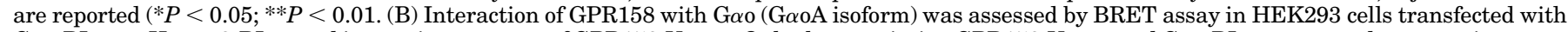

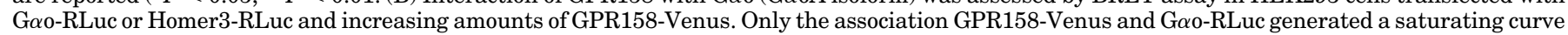

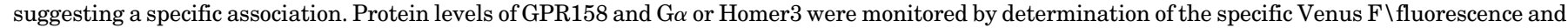

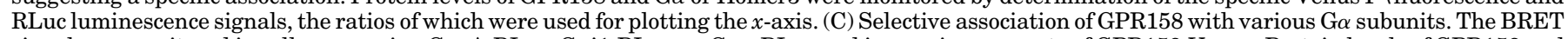

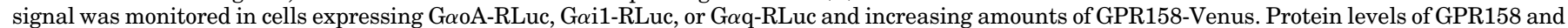

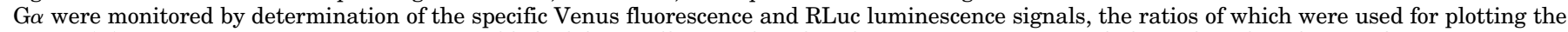

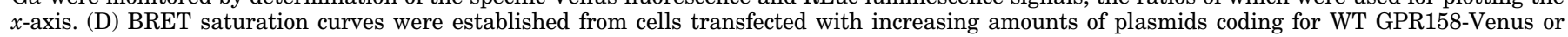

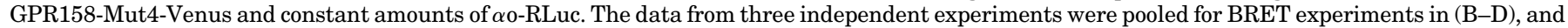
values are means \pm S.E.M. of triplicate determinations.

vasopressin receptor V2, known not to couple to Go (Fig. 6A). In addition, a basal coupling to Go of both MOR (Fig. 6A) and GABA $_{\mathrm{B}}$ (Fig. 6B) in the absence of ligand could be detected, but not for V2 (Fig. 6A). Moreover, the basal Go coupling of MOR increased as a function of MOR cell-surface protein level measured using nonpermeable fluorescent labeling of Snap tag domains inserted at the $\mathrm{N}$-terminal end of the receptor (Fig. 6C). Increasing GPR158 expression also led to a proportional decrease of BRET signal (Fig. 6C), suggesting a ligand-independent basal coupling of GPR158 to Go.

This GPR158 basal coupling to G protein was not, however, associated with any change in a second-messenger production-based readout (Fig. 6D), in contrast to what was observed with either MOR or GABA . The chimeric GqTop protein-containing portions of $\mathrm{Gi} / \mathrm{o}$ protein was used to allow $\mathrm{Gi} / \mathrm{o}$-coupled receptors to activate PLC, leading to the production of IP1, as illustrated with MOR or GABA $_{B}$ (Fig. 6D; Supplemental Fig. 5). In contrast to the large basal increase in IP1 production measured in $\mathrm{GABA}_{\mathrm{B}}$ receptor-expressing cells or, to a lesser extent, in MOR-expressing cells, no effect was observed with GPR158, despite a similar protein level of both receptors (Fig. 6D). These data bring no evidence for a constitutive canonical GqTop protein activation by GPR158, suggesting no activation of constitutive $\mathrm{Gi} / \mathrm{o}$ or $\mathrm{Gq}$ proteins 
A

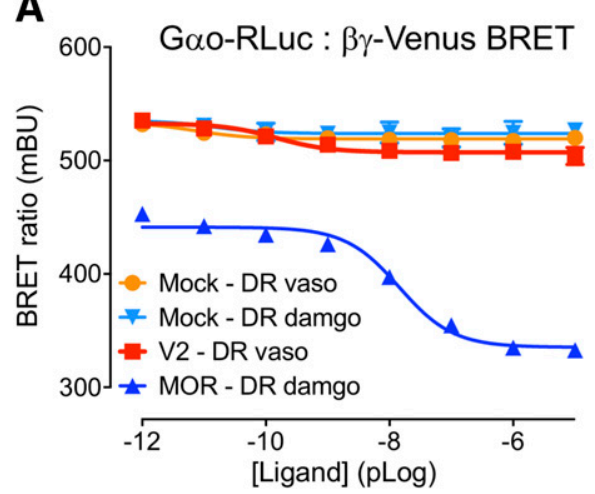

C

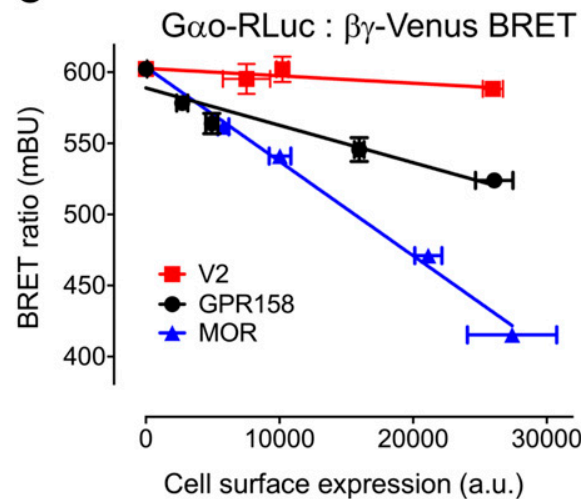

B

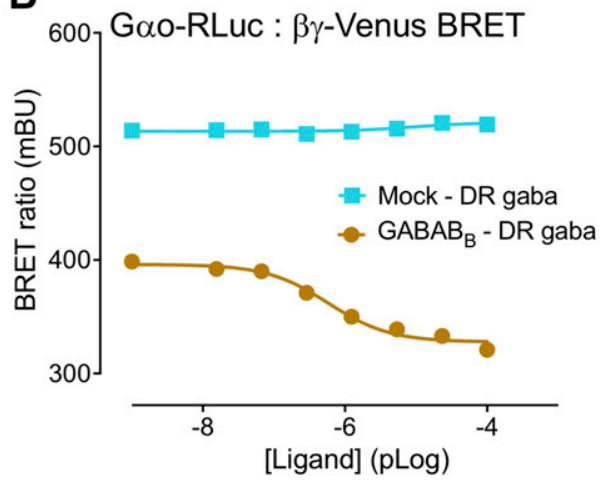

D

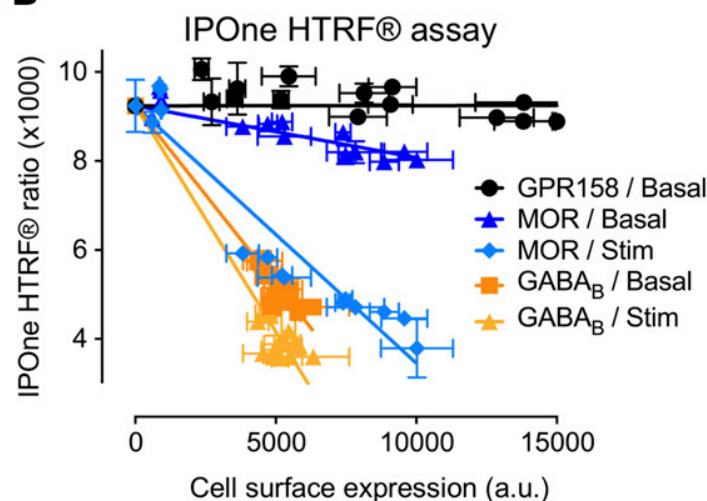

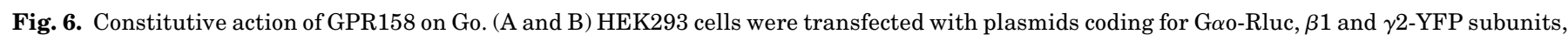

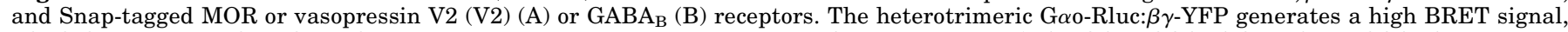

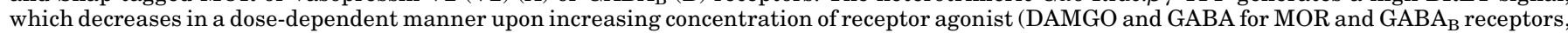

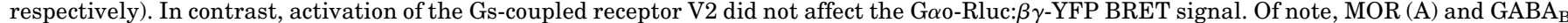

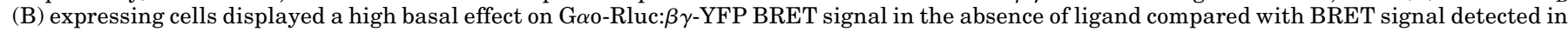

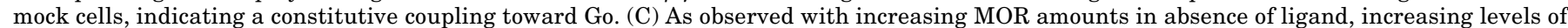

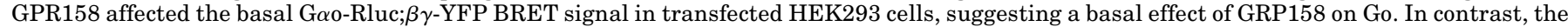

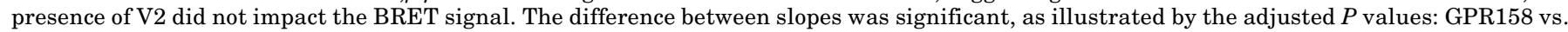

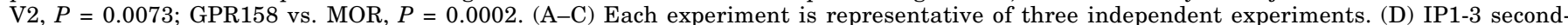

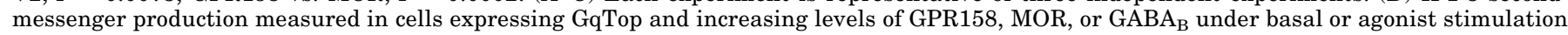

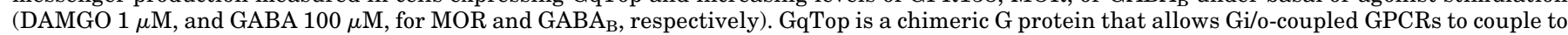

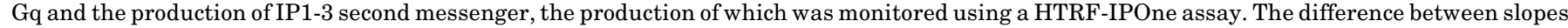

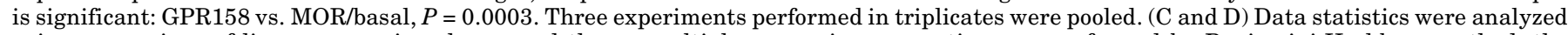

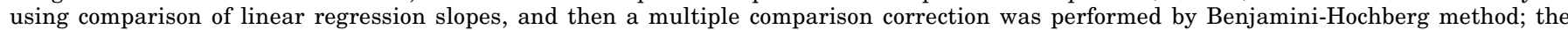
adjusted $P$ values are reported.

either. Moreover, no detectable GPR158 constitutive coupling to $\mathrm{Gs}$ or $\mathrm{Gi}$, as assessed by the adenylyl cyclase-driven production of the second-messenger cAMP (Supplemental Fig. 5), could be detected.

Further analysis of GPR158 7TM amino-acid sequence identified residues K502 and R505 in TM3 (Supplemental Fig. 6), highly conserved in class $\mathrm{C}$ receptors from fishes to humans and the mutations of which (such as mutations equivalent to K502E or R505A of GPR158 in $\mathrm{GABA}_{\mathrm{B}}$ receptor) do affect the ligand-induced and constitutive activity of $\mathrm{GABA}_{\mathrm{B}}$, mGlu1, or calcium-sensing GPCRs (Francesconi and Duvoisin, 1998; Ango et al., 2001; Duthey et al., 2002; Pin et al., 2004; Binet et al., 2007; Rondard et al., 2011). Surprisingly, the effect of GPR158 on Go activation/dissociation was not significantly altered when the receptor bore mutations K502E or R505A (Fig. 7, A and B). The preceding data showed that GPR158 affects the heterotrimeric G $\alpha 0-\beta \gamma$ association state and that this effect is unlikely due to a ligand-independent canonical coupling to Go protein. As additional evidence in support of this conclusion, the effect of GPR158 on Go is slightly diminished after treatment with the Gi/o inhibitor pertussis toxin (PTX), but the PTX effect is similar to that observed with mock-transfected cells, suggesting that the effect of GPR158 on Go is PTX-independent. In contrast, both the basal and agonist-induced Go activation observed with MOR is, as expected, largely inhibited by PTX (Fig. 7C).

GPR158 VCPWE Motifs Constitutively Increased Dissociated Go Levels in Cells Independently to Classic G-Protein Coupling. We found that the VCPWE motifs are essential for the GPR158 effect on Go. First, deletion of the C-terminal domain of GPR158 (GPR158$\Delta \mathrm{C} 1)$ completely abolished the GPR158-induced change in the Go BRET signal (Fig. 8A). Second, the mutation of all three motifs (GPR158-Mut4) suppressed the effect of GPR158 on the Go BRET sensor (Fig. 8B); however, the mutation of only one of these motifs was not sufficient to suppress the GPR158 effect (Fig. 8, C and D) or the combined mutation of motifs 1 and 2 or 2 and 3 (Fig. 8, E and F). Indeed, only the combined mutation of both VCPWE motifs 1 and 3 led to a suppression of the GPR158 effect on the Go BRET sensor (Fig. 8E). 
A

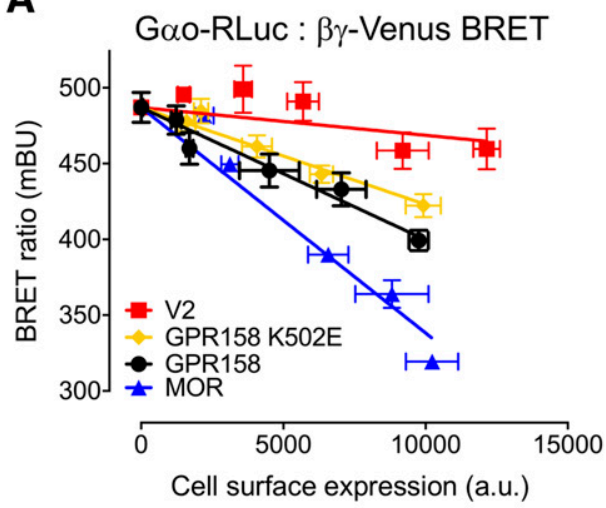

C

\section{Goo-RLuc : $\beta \gamma$-Venus BRET}

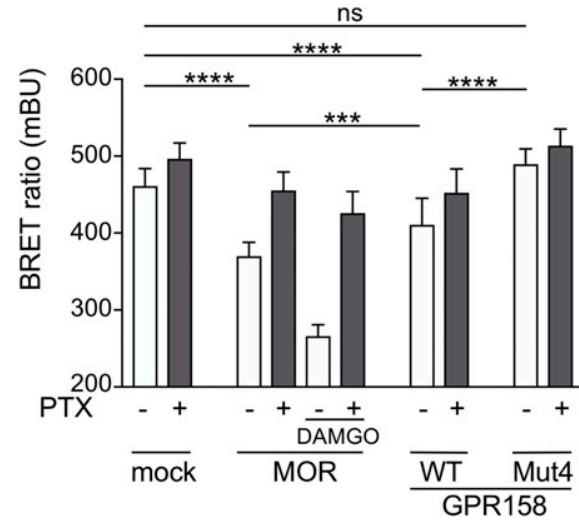

B

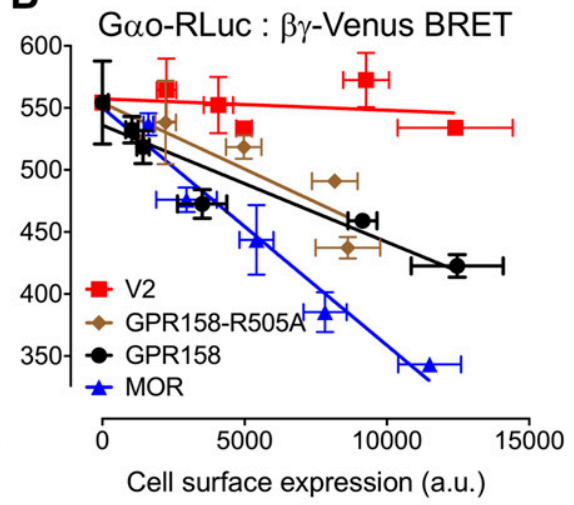

Fig. 7. GPR158-mediated decrease in G $\alpha$ o-Rluc: $\beta \gamma$-YFP BRET signal is unlikely due to a canonical activation of Go. (A and B) G $\alpha 0-$ Rluc: $\beta \gamma$-YFP BRET signal as a function of increasing amount of V2 vasopressin (V2), $\mu$ Opioid (MOR), WT or mutated K502E (A) or R505A (B) GPR158. (A) There is no significant difference between slopes of GPR158 and GPR158 K502E $(P=0.2379)$, whereas the difference is significant for GPR158 vs. V2 $(P=0.0222)$ and GPR158 K502E vs. V2 $(P=$ 0.0379 ). (B) There is no significant difference between slopes of GPR158 and GPR158 R505A $(P=0.7786)$, whereas the difference is significant for GPR158 vs. V2 $(P=0.0219)$ and GPR158 R505A vs. V2 $(P=0.0231)$. These experiments are representative of three independent experiments. (A and B) Data statistics were analyzed using comparison of linear regression slopes, and then a multiple comparison correction was performed by Benjamini-Hochberg method; the adjusted $P$ values are reported. (C) G $\alpha 0$-Rluc: $\beta \gamma$-YFP BRET signal was measured in mock-transfected cells or cells expressing MOR (basal or with application of DAMGO), GPR158 or GPR158Mut4, under control condition (white bars) or after overnight PTX treatment $(100 \mathrm{ng} / \mathrm{ml}$, gray bars). Data are means \pm S.D. for experiments performed in triplicates and pooled. Data statistics were analyzed using a one-way analysis of variance (ANOVA) test (variance was checked with a Brown-Forsythe method), and a multiple comparison correction was then performed by Tukey's method; the adjusted $P$ values are reported. ns, nonsignificant difference, *** $P<0.005$; **** $P<0.001$.

\section{Discussion}

Signaling and functions of GPR158 remain poorly characterized, although it has been proposed to be involved in the effect of osteocalcin in the brain (Khrimian et al., 2017). The scaffolding ability of GPR158 to interact with RGS7 allows it to regulate Go signaling induced by neighboring receptors when expressed in the same cells (Orlandi et al., 2012). Besides, GPR15 8 binds G $\alpha 0$, but its ability to couple to Go is still unclear. Furthermore, although GPR158 possesses three conserved VCPWE motifs, their function is still not elucidated.

Here we addressed the role of scaffolding and signaling of GPR158. We show that RGS7 interacts in the proximal part of the C-terminal intracellular domain, whereas $\mathrm{G} \alpha \mathrm{o}$ interacts downstream of this site with two of the three VCPWE motifs. Despite its ability to interact with $\mathrm{G} \alpha$ o, we did not find any evidence for a canonical basal activation of this $G$ protein by GPR158. Instead, we propose that GPR158, under basal conditions, can regulate Go signaling by trapping the G $\alpha 0$ subunit, leaving $\beta \gamma$ to act on its effectors. Although our data were all obtained in a recombinant system, our analysis using various expression levels of the partners and the use of different tags, inserted at different location and various approaches, provides a good indication that what is reported here is likely also occurring in native systems. With that said, we cannot exclude that the described process can be further controlled by other partners not expressed in HEK293 cells. A possible canonical G-protein activation upon agonist binding to GPR158 cannot be excluded, as we did not examine this possibility in the present study.
We delineated the RGS7 binding site in the 714-764 region of GPR158 C-terminal domain, proximal to the 7TM domain (Fig. 3). This small region overlaps with the CD1 region defined by Orlandi et al. (2015) as containing a binding site for RGS7 and displaying homology with R7BP protein. Indeed, GPR158 and R7BP compete for interacting with RGS7. RGS7 is composed of the RGS, GGL, and DEP domains. The latter is proposed to interact with GPR158 (Orlandi et al., 2012), as well as other proteins, but no clear DEP-binding consensus sequence has been identified. As observed with R7BP, GPR158 also stabilizes the RGS7 protein, leading to an increase in RGS7 protein level (Fig. 4). Consistent with this observation in HEK293 cells, a decrease of RGS7 protein level has been reported in GPR158 KO mice, with diminution of the pool of RGS7 in the membrane fraction and relocation into the cytoplasm, as revealed by electron microscopy in native and transfected models (Orlandi et al., 2012, 2015). Accordingly, GPR158 appears to regulate the pool of RGS7 and tunes its localization to the plasma membrane. RGS7 protein level is also known to depend on the coexpression of $\beta 5$ (Supplemental Fig. 1) (Anderson et al., 2009), which binds to RGS7 GGL domain to form a putative G-protein $\beta \gamma$ complex. As such, RGS7 binds GPR158 and $\beta 5$ via two independent domains, DEP and GGL, respectively, leaving its RGS domain free to bind active $\mathrm{G} \alpha \mathrm{o}$ and deactivate it.

We also demonstrated that two of the VCPWE motifs (i.e., motifs 1 and 3) of GPR158 were important for G $\alpha$ o binding, even in the absence of RGS7 (Fig. 5). Motif 2 in primates, including human GPR158, does not contain the conserved 
A

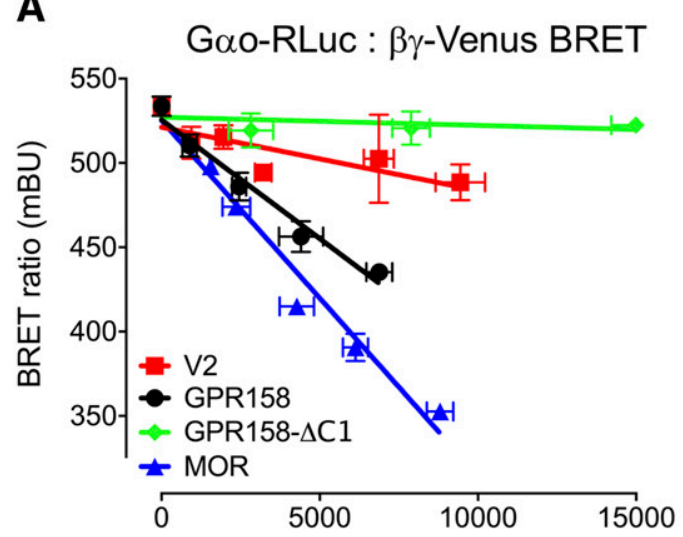

C

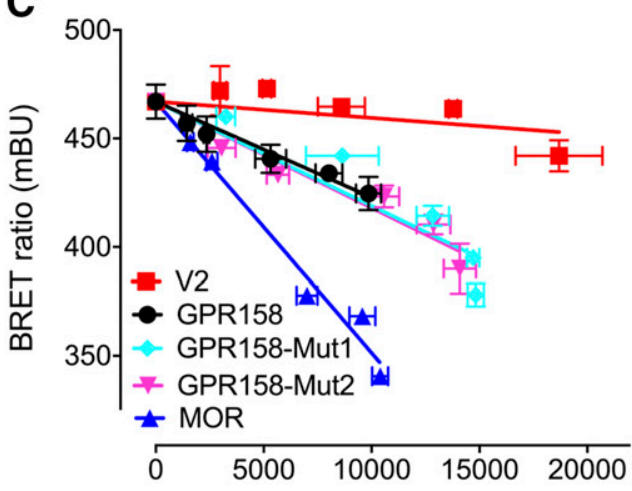

E

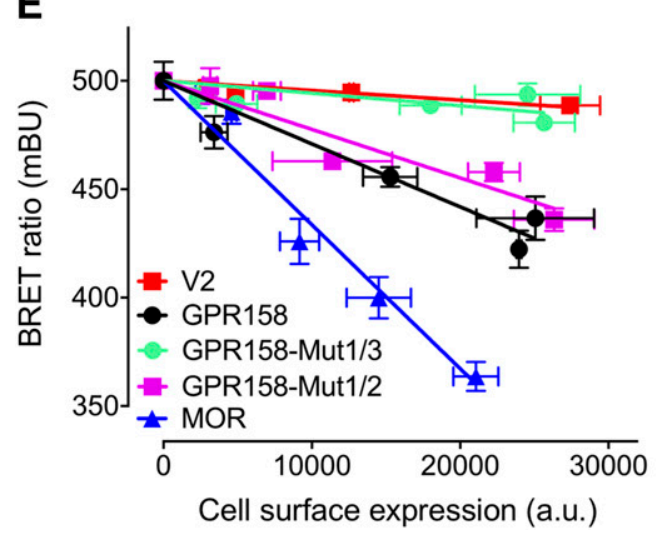

B

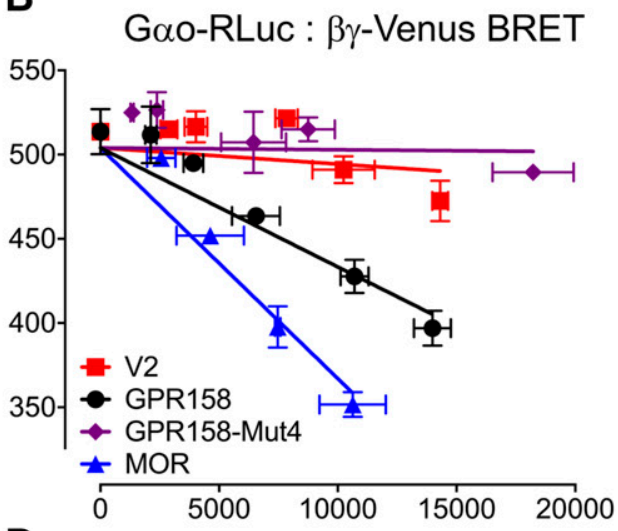

D

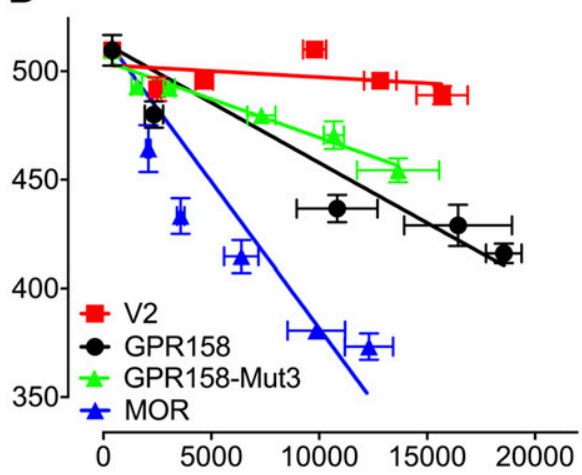

F

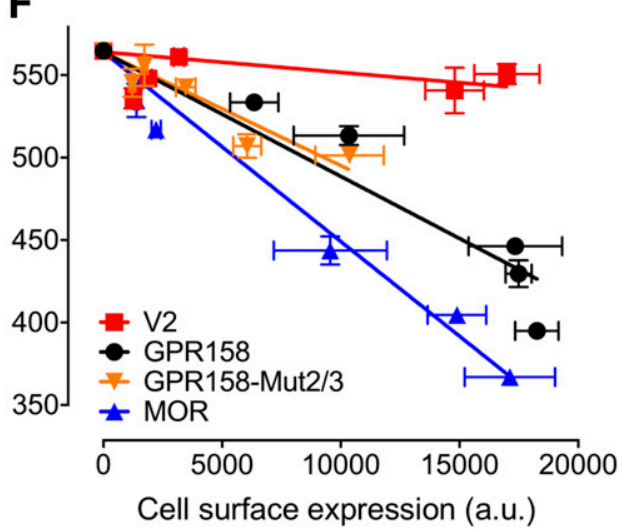

Fig. 8. Mutation of both VCPWE motifs 1 and 3 is required for suppressing GPR 158 constitutive action on Go. Variation of the G $\alpha$-Rluc: $\beta \gamma$-YFP BRET signal was measured in HEK293 cells expressing an increasing amount of V2, MOR, WT GPR158 (A-F), or GPR158- $\Delta$ C1 (A), -Mut4 (B), -Mut1 or -Mut2

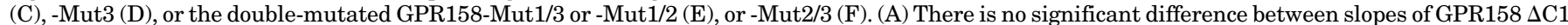

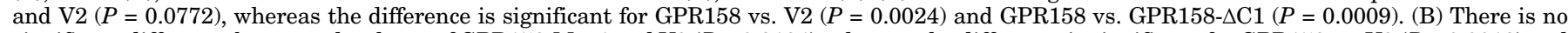

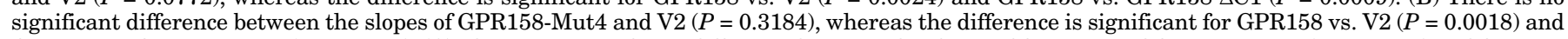

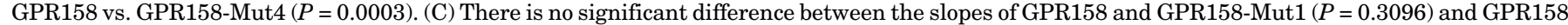

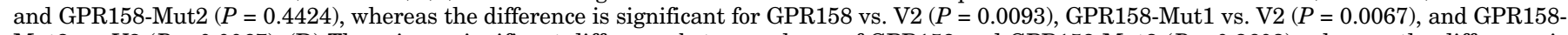

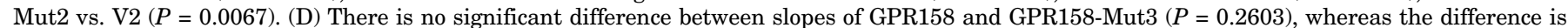

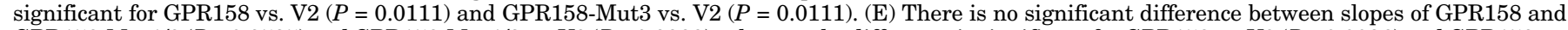

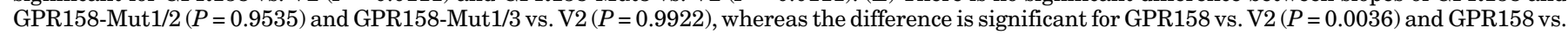

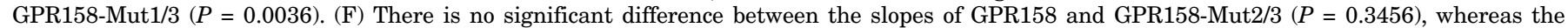

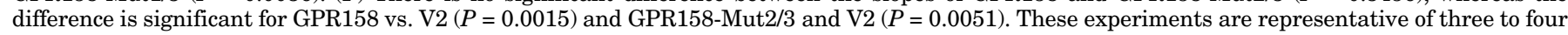

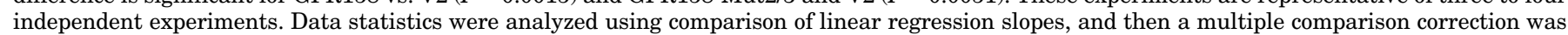
performed by Benjamini-Hochberg method; adjusted $P$ values are reported.

proline residue, suggesting it has lost its ability to bind G $\alpha 0$ during evolution (Supplemental Fig. 2) (Slep et al., 2001). Mutation of the three motifs did not completely suppress G $\alpha$ o binding, as revealed with the BRET approach, suggesting that there might be another site. Interestingly, Orlandi et al. (2015) reported two G $\alpha$ o binding sites in GPR158 C-terminal domain, one close to the RGS7 binding site and another one in the distal part of the C-terminal domain. Thus, according to our data, VCPWE motifs may correspond to this second distal site, whereas the other corresponds to the proximal site. The remaining G $\alpha$ o interaction observed after mutating the VCPWE motifs could also simply be indirect owing to a 
proximity to GPR158 resulting from G $\alpha$ o association with RGS7 or other proteins or GPCRs interacting with GPR158.

We further showed that the VCPWE motifs are not only important for $\mathrm{G} \alpha$ o binding but also impact $\mathrm{G} \alpha 0-\beta \gamma$ association. The observed decreased of BRET between Goo-RLuc and $\beta \gamma$-Venus in the presence of GPR158 (Fig. 6) could reflect the dissociation or the conformational change usually observed upon G-protein activation (Gales et al., 2006). Because we obtained no evidence for a canonical activation of $\mathrm{G}$ proteins by GPR158 in absence of ligand, however, we favored alternative hypotheses. The VCPWE motifs may bind G $\alpha$ o-RLuc, reducing its association with $\beta \gamma$-Venus, or G $\alpha 0-\beta \gamma$ complexes activated by other endogenous GPCRs may have led to the release of G $\alpha$ o, which can be trapped by VCPWE motifs, preventing their reassociation with $\beta \gamma$. Both situations likely occur, as the first explanation is supported by the PTX-insensitive component of the GPR158 effect on Go BRET sensor, whereas the second is supported by known action of VCPWE-related motifs. Indeed: 1) the ICPWE motif of the $\gamma$ subunit of the retinal PDE binds the active $\alpha$ transducin subunit (Slep et al., 2001), and 2) the distal site in the GPR158 C-terminal domain has been proposed to preferentially bind an active form of $\mathrm{G} \alpha \mathrm{o}$ (Orlandi et al., 2015). Taken together, these findings suggests that VCPWE motifs likely trap isolated $\mathrm{G} \alpha 0$ and inhibit them from activating their effectors while leaving $\beta \gamma$ free to activate its own effectors. GPR158 would then induce a ligand-independent signaling bias of $\beta \gamma$ versus $\alpha$ subunits, an effect reminiscent of the function of some group II AGS proteins (Blumer and Lanier, 2014). Of note, two VCPWE motifs (1 and 3) are required for this effect (Fig. 8), suggesting that either one Go binds to both motifs or, alternatively, that each motif binds one Go independently of each other, possibly differently regulated by RGS7. Further experiments will be necessary to clarify this point. Because GPR158 associates with Cav2 calcium channel in the rat brain (Müller et al., 2010) and Kv4.2 potassium channel in the mouse brain (Marionneau et al., 2009), which are both regulated by $\beta \gamma$ and RGS proteins, such a G $\alpha$ o-trapping mechanism by the VCPWE motifs may change the kinetics of such regulatory effects of GPR158. In the retina, where both RGS7 and the GPR158-related GPR179 containing 21 VCPWE motifs are expressed (Audo et al., 2012; Orlandi et al., 2012), such a mechanism could control the spatiotemporal regulation of signaling of photoreceptors and ON bipolar cells. This process may be reminiscent of the control of the PDE response by rhodopsin and transducin that involves RGS9/7 and the ICPWE motif of PDE $\gamma$ subunit (Slep et al., 2001).

Many GPCRs display constitutive activity in absence of ligand, leading to constitutive canonical G-protein coupling, and mutation-driven constitutive activity of some GPCRs leads to various diseases (Tao, 2008). GPR158 possesses class C GPCR features required for G-protein coupling (Bjarnadóttir et al., 2005), like lysine and arginine residues in TM3 previously shown to be important for G-protein coupling in GABA $_{\mathrm{B}}$ (Galvez et al., 2001; Binet et al., 2007) and mGlu5 (Doré et al., 2014; Koehl et al., 2019); however, we did not detect any constitutive G-protein activation when measuring Go/i, Gq, or Gs activity in HEK293 cells expressing WT or mutated GPR158. One can envisage that GPR158 displays no constitutive canonical G-protein coupling or that GPR158 couples to other pathways that have not been addressed in this work, like those resulting from G12/13 protein or the
G-independent arrestin pathway, although no direct coupling to arrestin has been clearly demonstrated for any class $\mathrm{C}$ GPCRs so far (Pin and Bettler, 2016). Another possibility is that GPR158 needs a GPCR partner, like the GABA $_{B}$ receptor, requiring the association of two different proteins GB1 and GB2 (Pin et al., 2004; Rondard et al., 2011). More work is needed to clarify this important issue. As mentioned, such data do not exclude a direct G-protein activation by GPR158 upon agonist binding.

Dimer formation is required for activation of the multidomain class $\mathrm{C}$ mGlu, calcium sensing, taste $\mathrm{T} 1 \mathrm{Rs}$, and $\mathrm{GABA}_{\mathrm{B}}$ receptors. Indeed, the intersubunit movement of the extracellular domain (ECD) resulting from ligand binding changes the interaction mode of the 7TM domains, leading to the activation of one of them (Xue et al., 2015; Pin and Bettler, 2016; Koehl et al., 2019). We have shown here that GPR158 also forms homodimers, making possible a similar activation process with ligands interacting in the GPR158 ECD; however, not only does this ECD not share similarity with that of other class C GPCRs, but also with any other protein of known structure, making it impossible to predict the mode of action of such a domain. It is clearly important to elucidate whether and how GPR158 can directly activate $\mathrm{G}$ protein upon activation with a ligand.

Not all 7TM proteins couple to G proteins, like the adiponectin receptor (Vasiliauskaité-Brooks et al., 2017), the GB1 subunit of $\mathrm{GABA}_{\mathrm{B}}$, or both T1R1 and T1R2, which need to be associated with T1R3 to form the umami and sweet taste receptors, respectively (Kniazeff et al., 2011). Some orphan GPCRs are also considered regulatory associated proteins that control the activity of functional GPCRs, as shown elegantly for the orphan receptor GPR50 that inhibits the melatonin receptor MT1 (Levoye et al., 2006) or controls TGF $\beta$ signaling (Wojciech et al., 2018). A recent article proposed that GRP158 mediates the action of the hormone osteocalcin (Khrimian et al., 2017), which has also been reported to activate GPRC6A, another class C GPCR (Pi et al., 2005). In cells deleted of GPR158, osteocalcin did not trigger an increase in brain-derived neurotropic factor expression (Khrimian et al., 2017), whereas the production of secondmessenger IP3 was decreased, suggesting that osteocalcin action on GPR158 modulates IP3 production. Although we did not observe any $\mathrm{G} \alpha \mathrm{q}$ binding or constitutive activity toward IP3 production in transfected HEK293 cells, this does not exclude a ligand-induced activation of the Gq pathway by osteocalcin. A signaling-partner protein may be missing in HEK293 cells to allow GPR158 to couple to Gq, as illustrated by the class $\mathrm{C}$ mGlu7 glutamate receptor, which needs to interact with Pick1 to couple to the PLC-IP3 pathway in neurons (Perroy et al., 2000). Moreover, mGlu4, reported to be $\mathrm{Gi} / \mathrm{o}-c o u p l e d$ in the recombinant system, is endogenously coupled to the Gq pathway in parallel fiber-Purkinje cell synapses (Abitbol et al., 2012).

According to our data and previous studies, GPR158 behaves as a scaffolding platform that tunes the Go pathway in an original way. In the absence of ligand, GPR158 displays various roles. First, it associates, stabilizes, and brings RGS7 to the plasma membrane, where RGS7 deactivates Go proteins activated by surrounding receptors (Orlandi et al., 2012). Second, the GPR158 VCPWE motifs-mediated trapping action on $\mathrm{G} \alpha \mathrm{o}$ could impact the Go signaling in the surrounding microenvironment and favor $\beta \gamma$-mediated signaling. Third, 
GPR158 can be associated with both RGS7 and G $\alpha$ o leading to a complex regulation of Go pathway involving VCPWE motifs. Finally, in the presence of ligand, GPR158 could couple to IP3 production via $\mathrm{Gq}$, as recently proposed (Khrimian et al., 2017). Integration of the signaling functions of GPR158 is a fascinating issue, as GPR158 would then control the Gq pathway in a canonical way and modulate at the same time the Gi/o pathways in a completely atypical way, similarly to some group II AGS proteins. Taken together, our data further illustrate the numerous possible ways 7TM proteins use to control cell signaling.

\section{Acknowledgments}

The project was supported by the EIDOS Collaborative team IGF-Cisbio Bioassays (Codolet, France). M.H. was supported by a "Presidence Grant" from the University of Montpellier (France). The pharmacology, BRET, and FRET experiments were performed using the ARPEGE Pharmacology-Screening-Interactome platform facility (UMS Biocampus), Montpelier, France. We thank Robert Healey for reading and English editing of the manuscript.

\section{Authorship Contributions}

Participated in research design: Prézeau.

Conducted experiments: Hajj, De Vita, Vol, Renassia, Bologna, Brabet, Cazade.

Contributed new reagents or analytical tools: Blahos, Labesse.

Performed data analysis: Hajj, De Vita, Bologna, Pastore, Prézeau. Wrote or contributed to the writing of the manuscript: Hajj, Pin, Prézeau.

\section{References}

Abitbol K, McLean H, Bessiron T, and Daniel H (2012) A new signalling pathway for parallel fibre presynaptic type 4 metabotropic glutamate receptors (mGluR4) in the rat cerebellar cortex. J Physiol 590:2977-2994

Anderson GR, Posokhova E, and Martemyanov KA (2009) The R7 RGS protein family: multi-subunit regulators of neuronal $\mathrm{G}$ protein signaling. Cell Biochem Biophys 54:33-46.

Ango F, Prézeau L, Muller T, Tu JC, Xiao B, Worley PF, Pin JP, Bockaert J, and Fagni L (2001) Agonist-independent activation of metabotropic glutamate receptors by the intracellular protein Homer. Nature 411:962-965.

Audo I, Bujakowska K, Orhan E, Poloschek CM, Defoort-Dhellemmes S, Drumare I, Kohl S, Luu TD, Lecompte O, Zrenner E, et al. (2012) Whole-exome sequencing identifies mutations in GPR179 leading to autosomal-recessive complete congenital stationary night blindness. Am J Hum Genet 90:321-330.

Ayoub MA, Maurel D, Binet V, Fink M, Prézeau L, Ansanay H, and Pin JP (2007) Real-time analysis of agonist-induced activation of protease-activated receptor 1/Galphai1 protein complex measured by bioluminescence resonance energy transfer in living cells. Mol Pharmacol 71:1329-1340.

Binet V, Duthey B, Lecaillon J, Vol C, Quoyer J, Labesse G, Pin JP, and Prézeau L (2007) Common structural requirements for heptahelical domain function in class A and class C G protein-coupled receptors. J Biol Chem 282:12154-12163.

Bjarnadóttir TK, Schiöth HB, and Fredriksson R (2005) The phylogenetic relationship of the glutamate and pheromone G-protein-coupled receptors in different vertebrate species. Ann N Y Acad Sci 1040:230-233.

Blumer JB and Lanier SM (2014) Activators of G protein signaling exhibit broad functionality and define a distinct core signaling triad. Mol Pharmacol 85:388-396.

Bockaert J, Perroy J, Bécamel C, Marin P, and Fagni L (2010) GPCR interacting proteins (GIPs) in the nervous system: roles in physiology and pathologies. Annu Rev Pharmacol Toxicol 50:89-109.

Brulé C, Perzo N, Joubert J-E, Sainsily X, Leduc R, Castel H, and Prézeau L (2014) Biased signaling regulates the pleiotropic effects of the urotensin II receptor to modulate its cellular behaviors. FASEB J 28:5148-5162.

Condomitti G, Wierda KD, Schroeder A, Rubio SE, Vennekens KM, Orlandi C, Martemyanov KA, Gounko NV, Savas JN, and de Wit J (2018) An input-specific orphan receptor GPR158-HSPG interaction organizes hippocampal mossy fiberCA3 synapses. Neuron 100:201-215.e9.

Doré AS, Okrasa K, Patel JC, Serrano-Vega M, Bennett K, Cooke RM, Errey JC, Jazayeri A, Khan S, Tehan B, et al. (2014) Structure of class C GPCR metabotropic glutamate receptor 5 transmembrane domain. Nature 511:557-562.

Doumazane E, Scholler P, Zwier JM, Trinquet E, Rondard P, and Pin J-P (2011) A new approach to analyze cell surface protein complexes reveals specific heterodimeric metabotropic glutamate receptors. FASEB J 25:66-77.

Duthey B, Caudron S, Perroy J, Bettler B, Fagni L, Pin JP, and Prézeau L (2002) A single subunit (GB2) is required for G-protein activation by the heterodimeric GABA(B) receptor. J Biol Chem 277:3236-3241.

El Moustaine D, Granier S, Doumazane E, Scholler P, Rahmeh R, Bron P, Mouillac B, Banères J-L, Rondard P, and Pin J-P (2012) Distinct roles of metabotropic glutamate receptor dimerization in agonist activation and G-protein coupling. Proc Natl Acad Sci USA 109:16342-16347.
Francesconi A and Duvoisin RM (1998) Role of the second and third intracellular loops of metabotropic glutamate receptors in mediating dual signal transduction activation. J Biol Chem 273:5615-5624.

Galés C, Van Durm JJ, Schaak S, Pontier S, Percherancier Y, Audet M, Paris H, and Bouvier M (2006) Probing the activation-promoted structural rearrangements in preassembled receptor-G protein complexes. Nat Struct Mol Biol 13:778-786.

Galvez T, Duthey B, Kniazeff J, Blahos J, Rovelli G, Bettler B, Prézeau L, and Pin JP (2001) Allosteric interactions between GB1 and GB2 subunits are required for optimal GABA(B) receptor function. EMBO J 20:2152-2159.

Gerber KJ, Squires KE, and Hepler JR (2016) Roles for regulator of G protein signaling proteins in synaptic signaling and plasticity. Mol Pharmacol 89:273-286.

Greif PA, Yaghmaie M, Konstandin NP, Ksienzyk B, Alimoghaddam K, Ghavamzadeh A, Hauser A, Graf A, Krebs S, Blum H, et al. (2011) Somatic mutations in acute promyelocytic leukemia (APL) identified by exome sequencing. Leukemia 25: $1519-1522$

Khrimian L, Obri A, Ramos-Brossier M, Rousseaud A, Moriceau S, Nicot A-S, Mera P, Kosmidis S, Karnavas T, Saudou F, et al. (2017) Gpr158 mediates osteocalcin's regulation of cognition. J Exp Med 214:2859-2873.

Kniazeff J, Prézeau L, Rondard P, Pin J-P, and Goudet C (2011) Dimers and beyond: the functional puzzles of class C GPCRs. Pharmacol Ther 130:9-25.

Kniazeff J, Saintot P-P, Goudet C, Liu J, Charnet A, Guillon G, and Pin J-P (2004) Locking the dimeric GABA(B) G-protein-coupled receptor in its active state. $J$ Neurosci 24:370-377.

Koehl A, Hu H, Feng D, Sun B, Zhang Y, Robertson MJ, Chu M, Kobilka TS, Laeremans T, Steyaert J, et al. (2019) Structural insights into the activation of metabotropic glutamate receptors. Nature 566:79-84

Levoye A, Dam J, Ayoub MA, Guillaume JL, Couturier C, Delagrange P, and Jockers R (2006) The orphan GPR50 receptor specifically inhibits MT1 melatonin receptor function through heterodimerization. EMBO J 25:3012-3023.

Marionneau C, LeDuc RD, Rohrs HW, Link AJ, Townsend RR, and Nerbonne JM (2009) Proteomic analyses of native brain K(V)4.2 channel complexes. Channels (Austin) 3:284-294

Maurel D, Comps-Agrar L, Brock C, Rives ML, Bourrier E, Ayoub MA, Bazin H, Tinel N, Durroux T, Prézeau L, et al. (2008) Cell-surface protein-protein interaction analysis with time-resolved FRET and snap-tag technologies: application to GPCR oligomerization. Nat Methods 5:561-567.

Maurel D, Kniazeff J, Mathis G, Trinquet E, Pin JP, and Ansanay H (2004) Cell surface detection of membrane protein interaction with homogeneous timeresolved fluorescence resonance energy transfer technology. Anal Biochem 329: $253-262$

Milligan G (2006) G-protein-coupled receptor heterodimers: pharmacology, function and relevance to drug discovery. Drug Discov Today 11:541-549.

Müller CS, Haupt A, Bildl W, Schindler J, Knaus HG, Meissner M, Rammner B, Striessnig J, Flockerzi V, Fakler B, et al. (2010) Quantitative proteomics of the Cav2 channel nano-environments in the mammalian brain. Proc Natl Acad Sci USA 107:14950-14957.

Oh DY, Kim K, Kwon HB, and Seong JY (2006) Cellular and molecular biology of orphan G protein-coupled receptors. Int Rev Cytol 252:163-218.

Orlandi C, Posokhova E, Masuho I, Ray TA, Hasan N, Gregg RG, and Martemyanov KA (2012) GPR158/179 regulate G protein signaling by controlling localization and activity of the RGS7 complexes. J Cell Biol 197:711-719.

Orlandi C, Xie K, Masuho I, Fajardo-Serrano A, Lujan R, and Martemyanov KA (2015) Orphan receptor GPR158 is an allosteric modulator of RGS7 catalytic activity with an essential role in dictating its expression and localization in the brain. $J$ Biol Chem 290:13622-13639.

Overington JP, Al-Lazikani B, and Hopkins AL (2006) How many drug targets are there?. Nat Rev Drug Discov 5:993-996.

Patel N, Itakura T, Gonzalez JM Jr, Schwartz SG, and Fini ME (2013) GPR158, an orphan member of G protein-coupled receptor Family C: glucocorticoid-stimulated expression and novel nuclear role. PLoS One 8:e57843.

Patel N, Itakura T, Jeong S, Liao C-P, Roy-Burman P, Zandi E, Groshen S, Pinski J, Coetzee GA, Gross ME, et al. (2015) Expression and functional role of orphan receptor GPR158 in prostate cancer growth and progression. PLoS One 10: e0117758.

Perroy J, Prezeau L, De Waard M, Shigemoto R, Bockaert J, and Fagni L (2000) Selective blockade of P/Q-type calcium channels by the metabotropic glutamate receptor type 7 involves a phospholipase C pathway in neurons. $J$ Neurosci 20 : 7896-7904

Pi M, Faber P, Ekema G, Jackson PD, Ting A, Wang N, Fontilla-Poole M, Mays RW, Brunden KR, Harrington JJ, et al. (2005) Identification of a novel extracellular cation-sensing G-protein-coupled receptor. J Biol Chem 280:40201-40209.

Pin J-P and Bettler B (2016) Organization and functions of $\mathrm{mGlu}$ and $\mathrm{GABA}_{\mathrm{B}}$ receptor complexes. Nature 540:60-68.

Pin JP, Kniazeff J, Goudet C, Bessis AS, Liu J, Galvez T, Acher F, Rondard P, and Prézeau L (2004) The activation mechanism of class-C G-protein coupled receptors. Biol Cell 96:335-342.

Prezeau L, Rives ML, Comps-Agrar L, Maurel D, Kniazeff J, and Pin JP (2010) Functional crosstalk between GPCRs: with or without oligomerization. Curr Opin Pharmacol 10:6-13.

Rives ML, Vol C, Fukazawa Y, Tinel N, Trinquet E, Ayoub MA, Shigemoto R, Pin JP, and Prézeau L (2009) Crosstalk between GABAB and mGlu1a receptors reveals new insight into GPCR signal integration. EMBO $J$ 28:2195-2208.

Rondard P, Goudet C, Kniazeff J, Pin JP, and Prézeau L (2011) The complexity of their activation mechanism opens new possibilities for the modulation of mGlu and GABAB class C G protein-coupled receptors. Neuropharmacology 60 $82-92$

Slep KC, Kercher MA, He W, Cowan CW, Wensel TG, and Sigler PB (2001) Structural determinants for regulation of phosphodiesterase by a $\mathrm{G}$ protein at $2.0 \mathrm{~A}$. Nature 409:1071-1077.

Tao YX (2008) Constitutive activation of G protein-coupled receptors and diseases: insights into mechanisms of activation and therapeutics. Pharmacol Ther 120:129-148. 
Thathiah A, Spittaels K, Hoffmann M, Staes M, Cohen A, Horré K, Vanbrabant M, Coun F, Baekelandt V, Delacourte A, et al. (2009) The orphan G protein-coupled receptor 3 modulates amyloid-beta peptide generation in neurons. Science 323: 946-951.

Vasiliauskaité-Brooks I, Sounier R, Rochaix P, Bellot G, Fortier M, Hoh F, De Colibus L, Bechara C, Saied EM, Arenz C, et al. (2017) Structural insights into adiponectin receptors suggest ceramidase activity. Nature 544:120-123.

Wojciech S, Ahmad R, Belaid-Choucair Z, Journé A-S, Gallet S, Dam J, Daulat A, Ndiaye-Lobry D, Lahuna O, Karamitri A, et al. (2018) The orphan GPR50 receptor promotes constitutive TGF $\beta$ receptor signaling and protects against cancer development. Nat Commun 9:1216.
Wood LD, Parsons DW, Jones S, Lin J, Sjöblom T, Leary RJ, Shen D, Boca SM, Barber T, Ptak J, et al. (2007) The genomic landscapes of human breast and colorectal cancers. Science 318:1108-1113.

Xue L, Rovira X, Scholler P, Zhao H, Liu J, Pin J-P, and Rondard P (2015) Major ligand-induced rearrangement of the heptahelical domain interface in a GPCR dimer. Nat Chem Biol 11:134-140.

Address correspondence to: Laurent Prézeau, Univ. Montpellier, IGF, 141, rue de la Cardonille, 34094 Montpellier, cedex 05, France. E-mail: Laurent.prezeau@ igf.cnrs.fr 